\title{
Unwrapped phase inversion with an exponential damping
}

\author{
Yunseok Choi ${ }^{1}$ and Tariq Alkhalifah ${ }^{1}$
}

\begin{abstract}
Full-waveform inversion (FWI) suffers from the phase wrapping (cycle skipping) problem when the frequency of data is not low enough. Unless we obtain a good initial velocity model, the phase wrapping problem in FWI causes a result corresponding to a local minimum, usually far away from the true solution, especially at depth. Thus, we have developed an inversion algorithm based on a space-domain unwrapped phase, and we also used exponential damping to mitigate the nonlinearity associated with the reflections. We construct the 2D phase residual map, which usually contains the wrapping discontinuities, especially if the model is complex and the frequency is high. We then unwrap the phase map and remove these cycle-based jumps. However, if the phase map has several residues, the unwrapping process becomes very complicated. We apply a strong exponential damping to the wavefield to eliminate much of the residues in the phase map, thus making the unwrapping process simple. We finally invert the unwrapped phases using the backpropagation algorithm to calculate the gradient. We progressively reduce the damping factor to obtain a high-resolution image. Numerical examples determined that the unwrapped phase inversion with a strong exponential damping generated convergent long-wavelength updates without low-frequency information. This model can be used as a good starting model for a subsequent inversion with a reduced damping, eventually leading to conventional waveform inversion.
\end{abstract}

\section{INTRODUCTION}

Full-waveform inversion (FWI) has been widely studied because theoretically it can provide detailed subsurface velocity structures. FWI, however, still suffers from the local minima problem keeping
FWI from converging to the global minimum. One of the major reasons for the local minima problem is the phase wrapping phenomena in the frequency domain or the cycle skipping that takes place in the time domain (Bunks et al., 1995; Virieux and Operto, 2009). Low-frequency information or a starting model close enough to the true model solves the phase wrapping problem in FWI. However, these preconditions are usually not available in practical cases. Therefore, there is a need to develop algorithms to unwrap the phases and invert the unwrapped phases.

One-dimensional phase unwrapping using temporal frequencies has been studied in the area of digital signal processing, in which the unwrapped phases are used to compute the complex cepstrum (Oppenheim and Schafer, 1975). One of the methods to unwrap phases using temporal frequencies is to estimate phase differences between adjacent frequencies in the phase spectrum of a signal, wrap the phase difference into a range of $-\pi$ and $+\pi$ by adding $\pm 2 \pi$, if the phase difference is out of the range, and add the phase differences from zero (or lowest) frequency (Oppenheim and Schafer, 1975). Another method is to compute the phase derivative with respect to the angular frequency and integrate it (Oppenheim and Schafer, 1975; Tribolet, 1977). More evolved methods factorize either the polynomials of the $z$-transform or the real component of it and use the zeros (roots) of the factorized function to unwrap the phase (McGowan and Kuc, 1982; Steiglitz and Dickinson, 1982; Treitel et al., 2006). These methods require low-frequency information and a small frequency interval along the frequency band to accurately unwrap the phases. Because frequency-domain FWI is performed for a single frequency at a time usually without low enough frequencies (Pratt et al., 1998; Shin and Min, 2006), the phase unwrapping using temporal frequencies does not fully address the cycle skipping issue in frequency-domain FWI.

On the other hand, the 2D (space variables) case has been tackled in the area of topography from synthetic aperture radar and optical interferometers (Ghiglia and Pritt, 1998). Two-dimensional phase unwrapping is processed on a phase map of 2D space coordinates for a single frequency using path-following methods or minimumnorm methods (Goldstein et al., 1988; Flynn, 1996; Ghiglia and

\footnotetext{
Manuscript received by the Editor 20 October 2014; revised manuscript received 3 April 2015; published online 28 July 2015.

${ }^{1}$ King Abdullah University of Science and Technology, Physical Science and Engineering Division, Thuwal, Saudi Arabia. E-mail: yunseok.choi@kaust.edu .sa; tariq.alkhalifah@kaust.edu.sa.

(C) 2015 Society of Exploration Geophysicists. All rights reserved.
} 
Pritt, 1998; Su and Chen, 2004). An important issue in 2D phase unwrapping is the existence of residues in a phase map. Residues in a phase map are detected when the evaluation of a simple closedpath integral of gradient of phase with respect to the space variable is not zero, where the sign of evaluation determines the charge of residue. Residues in a phase map are formed when the frequencydomain complex-valued data in 2D space are close to zero. If no residue exists in a phase map, 2D phase unwrapping is path independent and the task is trivial. However, in most practical cases, phase maps include many residues; thus, the phase unwrapping process is path dependent and becomes quite complicated, and the phase unwrapping results depend on the applied algorithms (Ghiglia and Pritt, 1998). Two-dimensional phase unwrapping has the potential to be applied to frequency-domain FWI because the 2D phase map of source and receiver coordinates for a single frequency can be obtained from the seismic survey.

The phase unwrapping issue in FWI has been tackled to overcome the local minima problem without the help of low-frequency information. Shah et al. (2012) construct a 2D phase map of source and receiver coordinates, and then unwrap the $2 \mathrm{D}$ phase map. They finally invert the unwrapped phases using the phase inversion algorithm of Shin and Min (2006). However, their method has a problem when the available minimum frequency of data is somewhat high, and the initial model is far from the true model, which causes many residues in the phase map. On the other hand, Choi and Alkhalifah $(2011,2013)$ estimate the phase derivative with respect to angular frequency, or what they refer to as the instantaneous traveltime, free from the wrapping phenomena and invert the phase derivative. When taking the derivative of phase with respect to the angular frequency, the phase information moves to the amplitude part of the resulting complex-valued quantity, which has no bound of range and thus no wrapping. They also incorporate exponential damping to eliminate the complexity of wavefield. Using a strong damping, the inversion of the phase derivative provides a long-wavelength structure without low-frequency information. However, calculation of the gradient of the objective function, required for the inversion
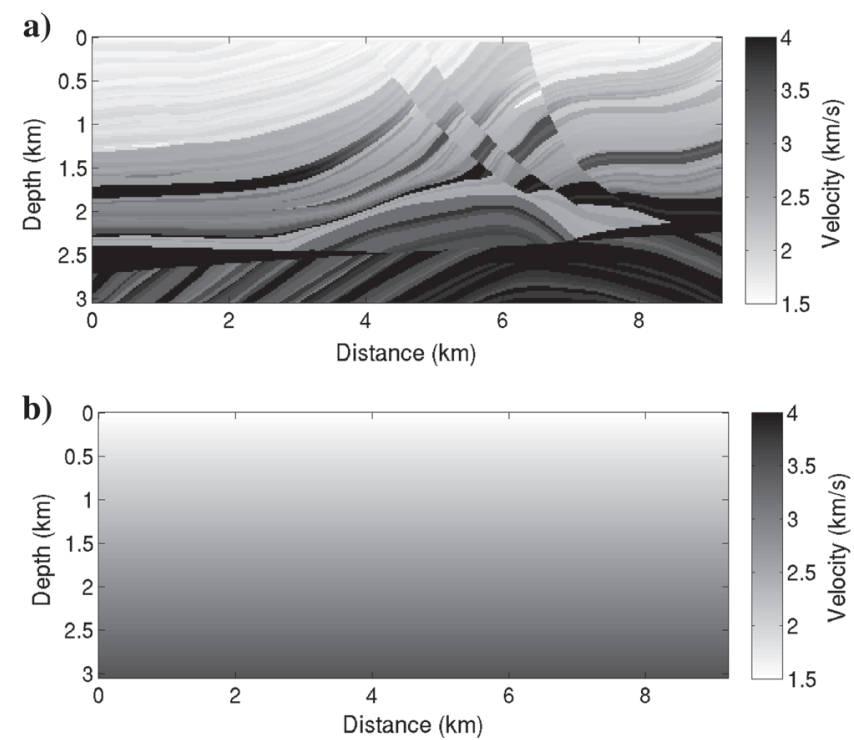

Figure 1. The (a) Marmousi velocity model and (b) a linearly increasing velocity model used as a starting model for inversions. of the phase derivative, is quite complicated and requires five modeling steps. Thus, its computational cost is much higher than that of conventional FWI.

In this paper, we construct a 2D phase residual map and then unwrap the phase map. Our algorithm is similar to that of Shah et al. (2012), but we also adopt an exponential damping to reduce the number of residues in a phase map. In the context of seismic exploration, a residue in a phase map is formed when multiple events interfere with each other; thus, the complex value of data in a phase map is close to zero at the position of interference. In other words, the number of residues in a phase map is related to the complexity of the wavefield (or the complexity of the subsurface velocity model). If there is a single event and no interference, the phase map has no residue. Because strong exponential damping suppresses latearrival events, the interference between multiple events (or the complexity of the wavefield) is removed; thus, residues in a phase map are eliminated even at a high frequency. Therefore, the 2D phase unwrapping process with a strong damping becomes path independent and provides a unique solution. We finally invert the unwrapped phases, in which the back-propagation algorithm is used to calculate the gradient, which is similar to conventional FWI except for the new residual vector. The inversion of the phase derivative of Choi and Alkhalifah $(2011,2013)$ also applies an exponential damping to the wavefield to eliminate the complexity of the data, but our inversion algorithm is cheaper than the inversion of the phase derivative because our inversion algorithm requires only two modeling steps and the cost of the unwrapping process is insignificant. We use the inverted velocity model as a new starting model for the inversion with a reduced damping and conventional FWI to obtain a higher resolution image.

In the following sections, we show several phase residual maps according to frequencies and damping factors. Then, we explain the inversion algorithm using the unwrapped phases. In the numerical examples, we apply the unwrapped phase inversion with an exponential damping to the Marmousi synthetic data and SEG 2014 benchmark data without low-frequency information to demonstrate the feasibility of our algorithm.

\section{THEORY}

In this section, we describe the construction of the phase residual map and its properties, and then we briefly introduce the unwrapping process. Last, we explain the inversion algorithm using the unwrapped phases.

\section{D phase residual map}

For the 2D phase unwrapping, we first need to construct a $2 \mathrm{D}$ phase map of the source and receiver coordinates. In this study, we consider only $2 \mathrm{D}$ seismic exploration. In the phase map, source and receiver positions share the same index number, which means the diagonal element indicates that the sources and receivers are located in the same position. In land seismic exploration, where receivers are fixed and only the source position moves, the phase map has elements on both sides of the diagonal line. On the other hand, in the case of marine seismic exploration, the phase map has elements on only one side of the diagonal line.

The modeled and observed data in the frequency domain at a certain frequency are expressed, respectively, as 


$$
\hat{u}_{i, j}=A_{i, j}^{u} \exp \left(i \theta_{i, j}^{u}\right) \quad \text { and } \quad \hat{d}_{i, j}=A_{i, j}^{d} \exp \left(i \theta_{i, j}^{d}\right)
$$

where subscripts $i$ and $j$ are the source and receiver index, superscripts $u$ and $d$ stand for the modeled and observed data, and $A$ and $\theta$ are the amplitude and phase of the data, respectively. We can calculate the phase residuals in two ways: one is to construct the phase maps separately for each of the modeled and observed data, and then we unwrap the two phase maps separately, and finally we estimate the difference between the two unwrapped phases for phase inversion. The other way is to construct the phase residual map $(\hat{u}-\hat{d})$ and then unwrap the phase map. We prefer the second way because it requires only one phase map and one unwrapping process, and also the phase residual map is usually simpler than the phase maps of the modeled or observed data when the initial (or inverted) model approaches the true model. If the damping is strong, the two approaches give the same result even if an initial model is far from the true model. From equation 1, we can estimate the phase residual as

a)

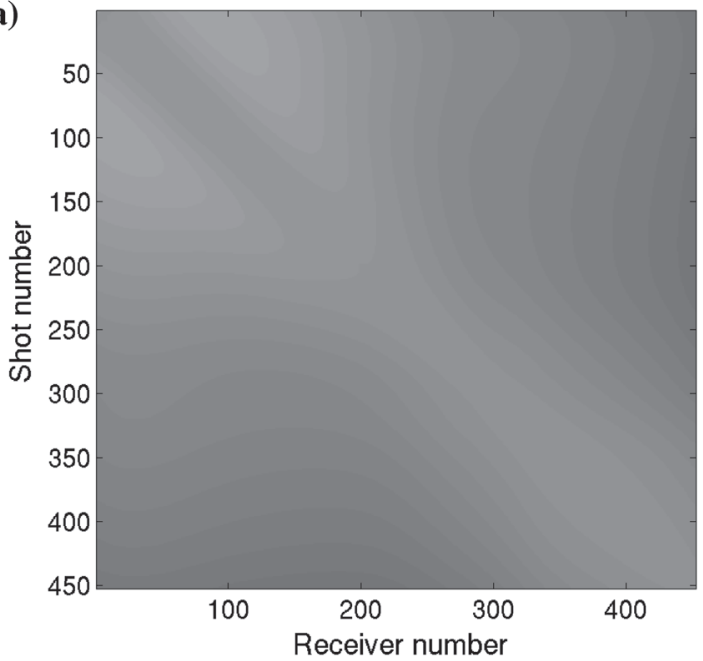

b)

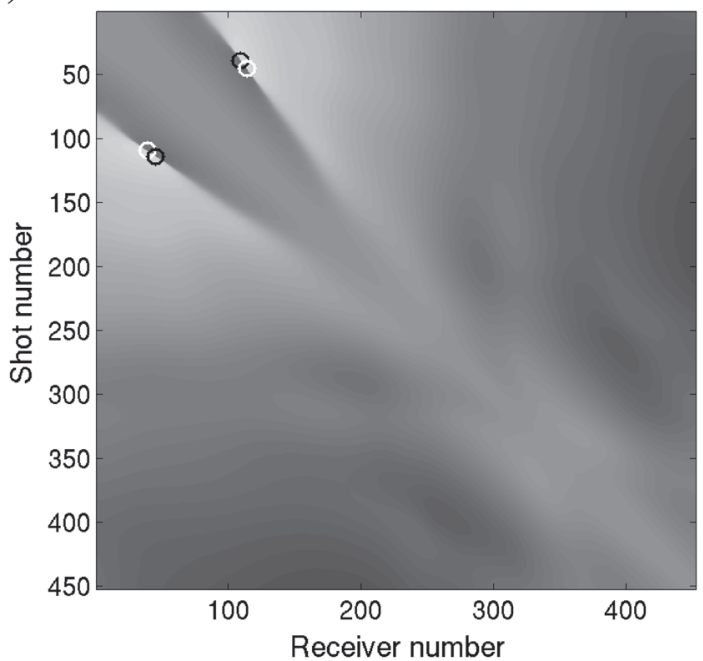

$$
\theta_{i, j}^{u}-\theta_{i, j}^{d}=\operatorname{Im}\left\{\operatorname{clog}\left(\frac{\hat{u}_{i, j}}{\hat{d}_{i, j}}\right)\right\}
$$

where "clog" indicates the complex logarithm, which is defined (Oppenheim and Schafer, 1975) as

$$
\operatorname{clog}\left(\hat{u}_{i, j}\right)=\log \left|\hat{u}_{i, j}\right|+i \operatorname{ARG}\left(\hat{u}_{i, j}\right),
$$

where $\operatorname{ARG}\left(\hat{u}_{i, j}\right)$ stands for the principal value of phase of $\hat{u}_{i, j}$. The phase residual $\left(\theta_{i, j}^{u}-\theta_{i, j}^{d}\right)$ in equation 2 always exists between $-\pi$ and $\pi$, and these bounds are the definition of the wrapping effect of the phase (or the jump of the phase).

Many phase unwrapping algorithms can be used to unwrap the phase map. However, if the phase map includes residues, the unwrapping process becomes very complicated. The existence of a residue in a region of the phase map is defined as cases in which the contour integral over the region is given as

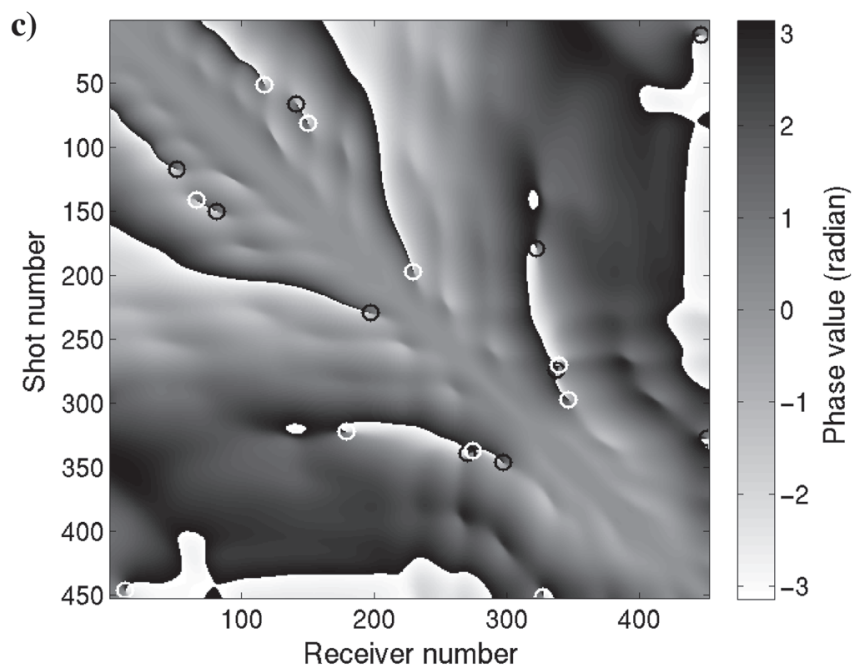

d)

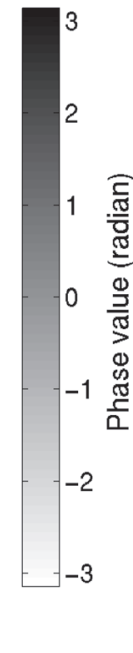

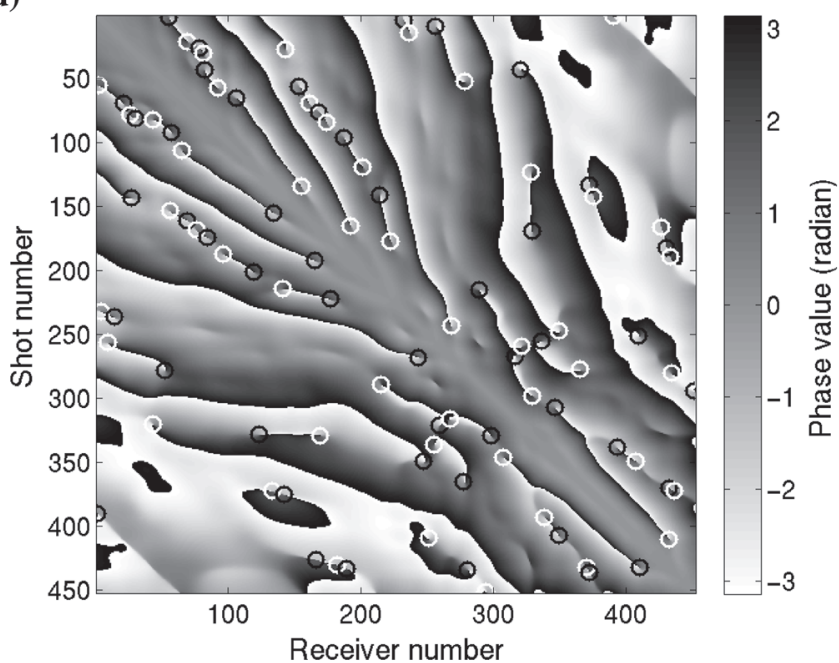

Figure 2. The phase residual maps of (a) 0.5 , (b) 1, (c) 2, and (d) $3 \mathrm{~Hz}$ with detected residues. The black and white circles indicate the detected plus and minus charge residues, respectively. 


$$
\oint \nabla \theta(\mathbf{r}) \cdot \mathbf{d r}=2 \pi m
$$

where $\nabla \theta=\partial \theta / \partial x \mathbf{i}+\partial \theta / \partial y \mathbf{j}, \mathbf{d r}=d x \mathbf{i}+d y \mathbf{j}, \mathbf{i}$ and $\mathbf{j}$ are the $x$ and $y$-direction unit vectors, and $m$ is the sum of the enclosed phase residue charges (Ghiglia and Pritt, 1998). Note that there are residues of plus charge $(+1)$ and minus charge $(-1)$. The residues in the phase map are located at the edge of the disconnected branch lines.

We construct and display examples of the phase residual map for several frequencies. We generate synthetic data (observed data) from the Marmousi model (Figure 1a) and modeled data from the linearly increasing velocity model (Figure 1b). We use a frequency-domain FEM modeling technique to generate the observed and modeled data. We place 452 receivers at a depth of $20 \mathrm{~m}$ with an interval of $20 \mathrm{~m}$ and shots at the same position of the receivers. We construct the phase residual maps according to equation 2 and detect residues in the phase map using equation 4 . Figure 2 shows the
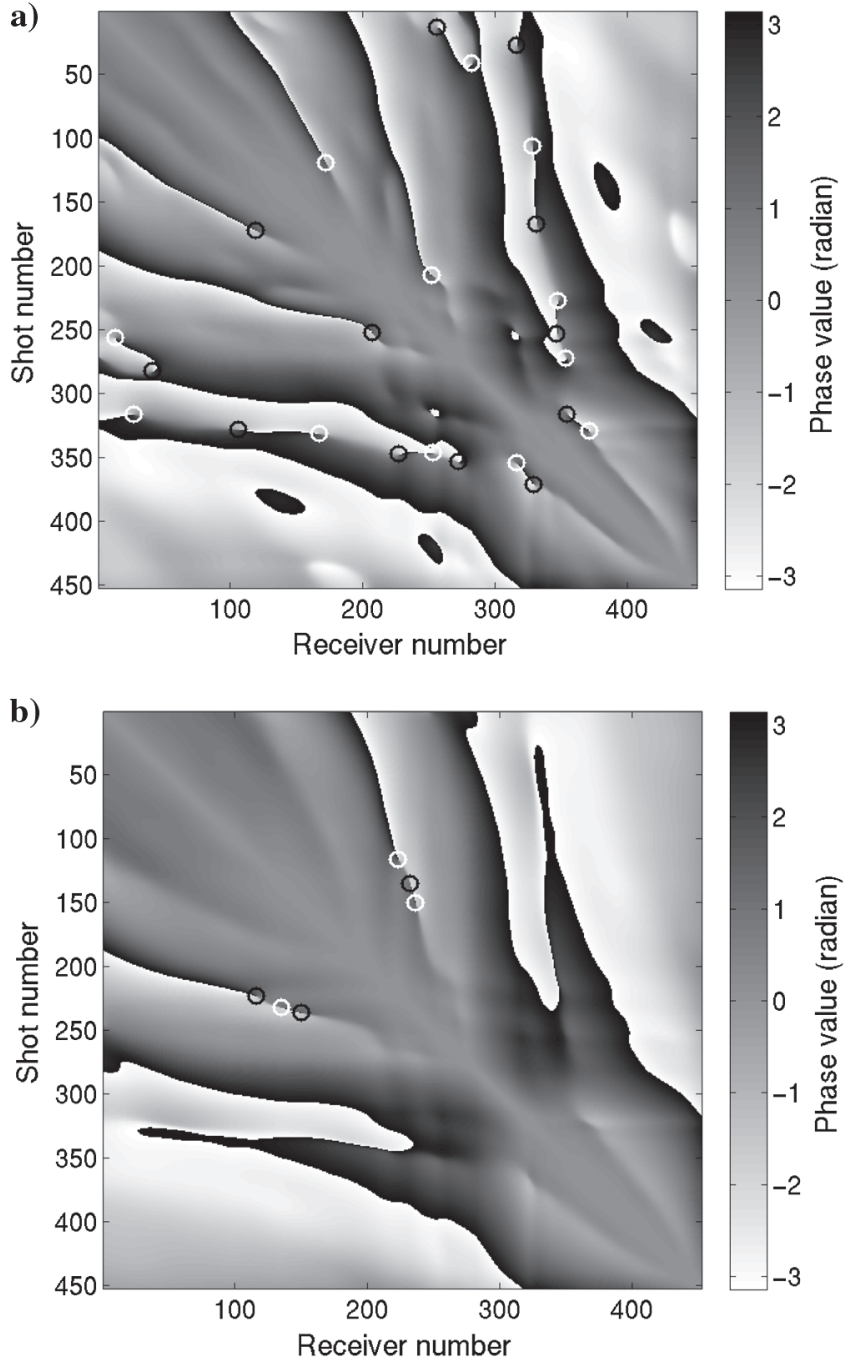

phase residual maps and the detected residues for several frequencies. There is no discontinuity (or wrapping) and no residue in the phase residual map at $0.5 \mathrm{~Hz}$ (Figure 2a), whereas the complexity of the phase and the number of residues increase as frequency increases (Figure 2b-2d). These phase wrapping phenomena lead to the local minima problem in FWI.

The available frequency of seismic data is usually not low enough for FWI; thus, the available phase residual map and its unwrapping process encounter the residue problem. To eliminate the residues in the phase map, in the next section we apply an exponential damping to the wavefield.

\section{Exponential damping for the phase map}

Exponential damping of wavefields has been applied to reduce the complexity of the data for FWI (Brenders and Pratt, 2007; Shin and Cha, 2008, 2009; Brossier et al., 2009). The damping term applied to the inversion has a trade-off between reducing the nonlinearity of inversion and losing the possibility to define a deeper
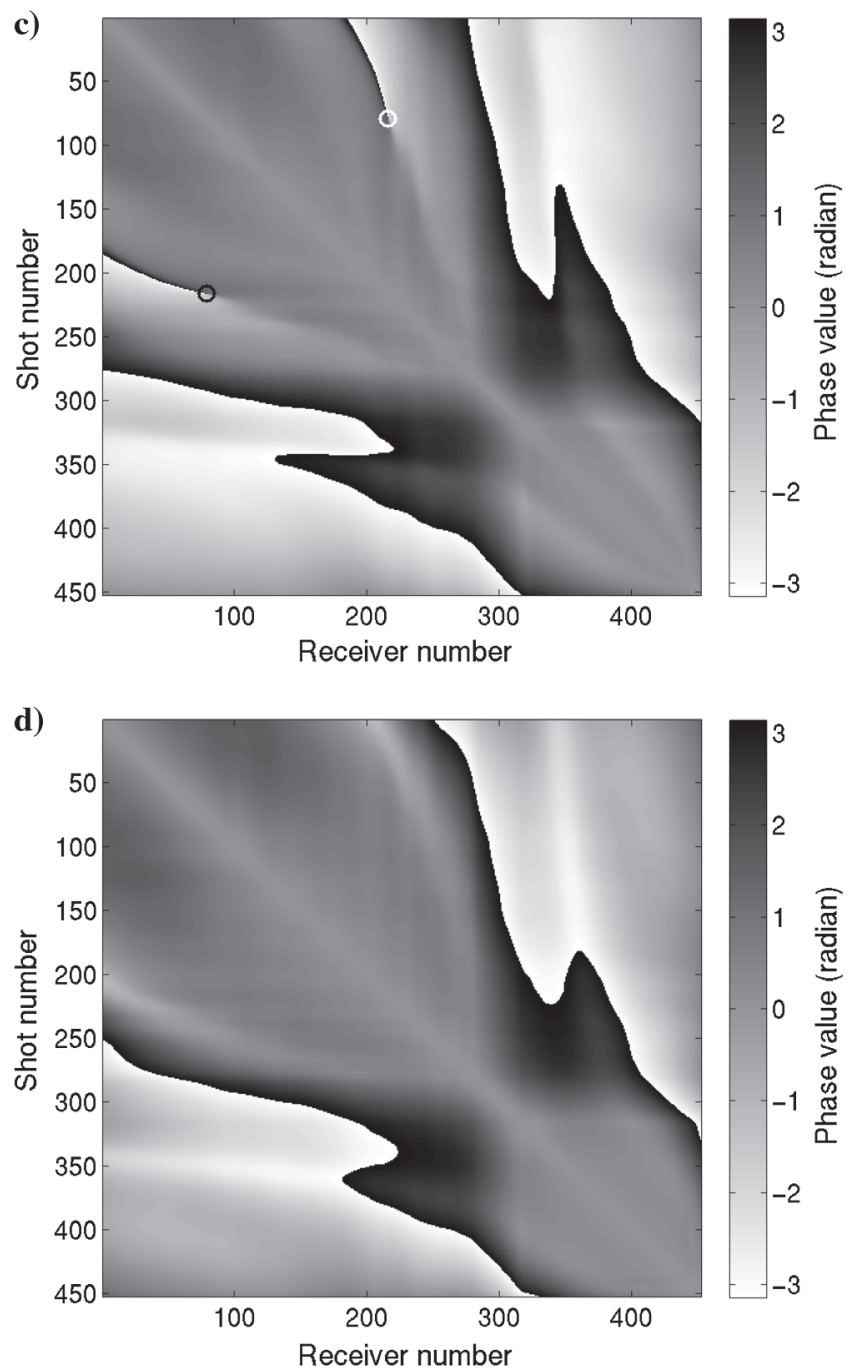

Figure 3. The phase residual maps at $3 \mathrm{~Hz}$ with damping factors of (a) 2, (b) 5, (c) 10, and (d) $30 \mathrm{~s}^{-1}$ with detected residues. The black and white circles indicate the detected plus and minus charge residues, respectively. 
structure. Because the damping term can reduce the complexity of the data, it can also reduce the residues in the phase map. In our study, we use exponential damping because it is easily implemented in frequency-domain modeling.

The exponential damped wavefield $(\bar{u}(t))$ is expressed as a multiplication of the original wavefield $(u(t))$ and exponential damping $\left(e^{-\alpha t}\right)$ as

$$
\bar{u}(t)=u(t) e^{-\alpha t},
$$

where $u(t)$ and $\bar{u}(t)$ are the causal functions and $\alpha$ is a damping factor. The Fourier transform of the damped wavefield is written as

$$
\hat{u}(\omega)=\int_{0}^{\infty} \bar{u}(t) e^{i \omega t} \mathrm{~d} t=\int_{0}^{\infty} u(t) e^{i(\omega+i \alpha) t} \mathrm{~d} t,
$$

where $\omega$ is an angular frequency. In the last term of equation 6 , the damping factor $\alpha$ is located in the imaginary part of the complex angular frequency. This means that frequency-domain modeling with a complex angular frequency generates an exponential- damped wavefield, where the imaginary part of the complex angular frequency plays a role of exponential damping (Shin et al., 2002; Min and Shin, 2006). The frequency-domain modeling technique using a complex angular frequency written as

$$
\hat{\mathbf{S}} \hat{\mathbf{u}}=\hat{\mathbf{f}},
$$

where $\hat{\mathbf{S}}$ is a modeling operator matrix using a complex angular frequency (Oh and Min, 2013), $\hat{\mathbf{u}}$ is a damped wavefield vector, and $\hat{\mathbf{f}}$ is a source vector with damping. The damped modeled wavefield $\hat{\mathbf{u}}$ is obtained by factorizing the matrix $\hat{\mathbf{S}}$ and the Gauss elimination algorithm.

On the other hand, the observed wavefield with exponential damping is obtained by the Fourier transform of the time-domain wavefield multiplied by an exponential damping, which is expressed as

$$
\hat{d}(\omega, \alpha)=F T[d(t) \exp (-\alpha t)]
$$
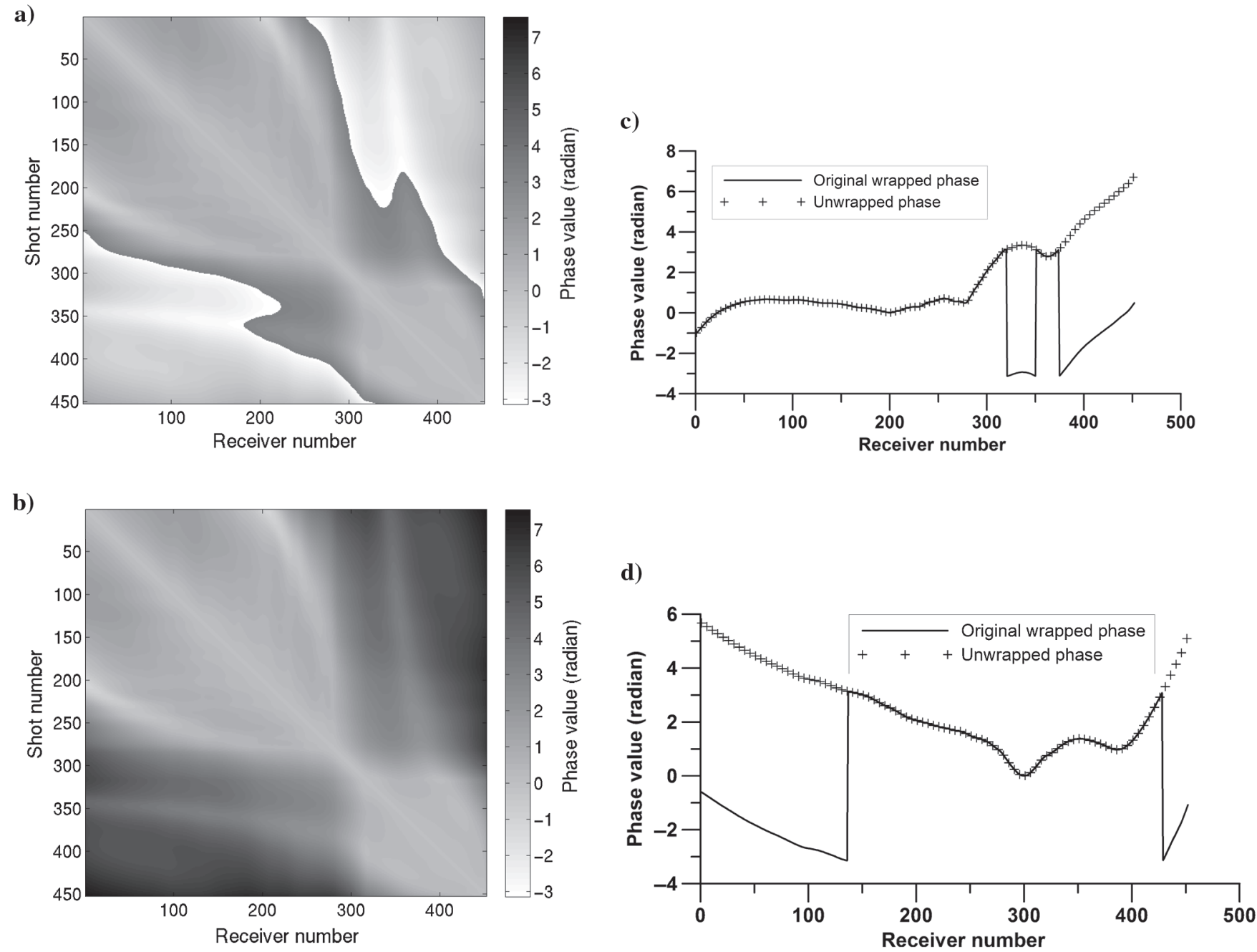

Figure 4. The (a) original phase residual map at $3 \mathrm{~Hz}$ with a damping factor of $30 \mathrm{~s}^{-1}$ and (b) its unwrapped version. The profiles of the original wrapped and unwrapped phase map are compared at shot numbers of (c) 200 and (d) 300. Figures 3d and 4a are the same phase map with different scale ranges. 
where $d(t)$ is the original time-domain observed data, $\alpha$ is the damping factor, and $t$ represents the time.

We estimate the phase residual of the damped wavefields using equation 2, and we detect residues in the phase maps. Figure 3 shows the phase residual maps and detected residues at $3 \mathrm{~Hz}$ with various damping factors $\left(2,5,10\right.$, and $\left.30 \mathrm{~s}^{-1}\right)$ using the same velocity models in Figure 1. Even the smallest damping factor $2 \mathrm{~s}^{-1}$ reduces the number of residues (Figure 3a) when comparing Figures $2 \mathrm{~d}$ and $3 \mathrm{a}$. The higher the damping factor is, the smaller is the number of residues in the phase residual map. With a high damping factor $\left(30 \mathrm{~s}^{-1}\right)$, we can obtain a phase residual map with no residues (Figure 3d), in which we can easily unwrap the phases.

Our strategy for selecting an appropriate damping factor for the first stage of unwrapped phase inversion is to detect residue points in the phase residual maps with several damping factors, following which we select the minimum damping factor for which the phase map has no residue. Detecting the residue in phase maps of several damping factors is not expensive compared with the FWI process, and it is needed only one time before the implementation of FWI as a preliminary setting.

Note that, once the residual map with strong damping has no residue and only simple branch lines (or discontinuous lines), we should unwrap the phases to avoid the local minima problem in FWI.

\section{Phase unwrapping process}

In this section, we describe the unwrapping process for the phase map free of residue. If the phase map has no residue, the unwrapping process is path independent and trivially simple (Ghiglia and Pritt, 1998). We first set a path line in the phase map that does not cross itself, estimate the phase differences between adjacent points on the path line, wrap the phase difference into a range of $-\pi$ and $+\pi$ by adding $\pm 2 \pi$ if the phase difference is out of the range, and sum the phase differences from the starting point of the path line (Itoh, 1982). In our study, we put the starting point on a diagonal
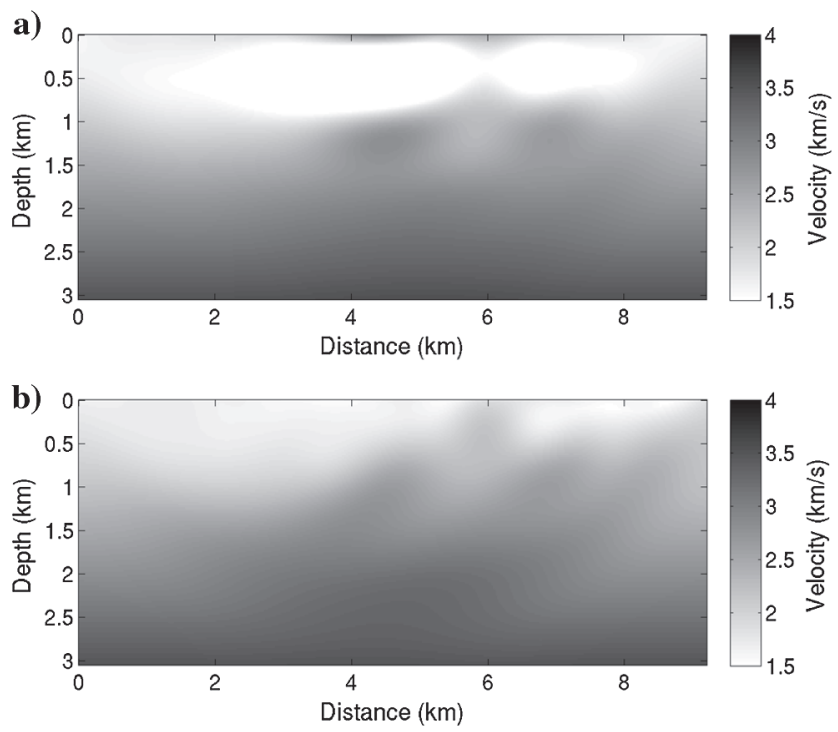

Figure 5. The inverted velocity model for a single frequency of $3 \mathrm{~Hz}$ at a damping factor of $30 \mathrm{~s}^{-1}$ by the (a) wrapped phase inversion and (b) unwrapped phase inversion. element in the phase map because the phase value (or phase residual) on the diagonal elements is regarded as the correct value. The ending point is put on the boundary element in the same vertical or horizontal line with the starting point. We trace many paths to cover the whole domain of the phase map, but these paths do
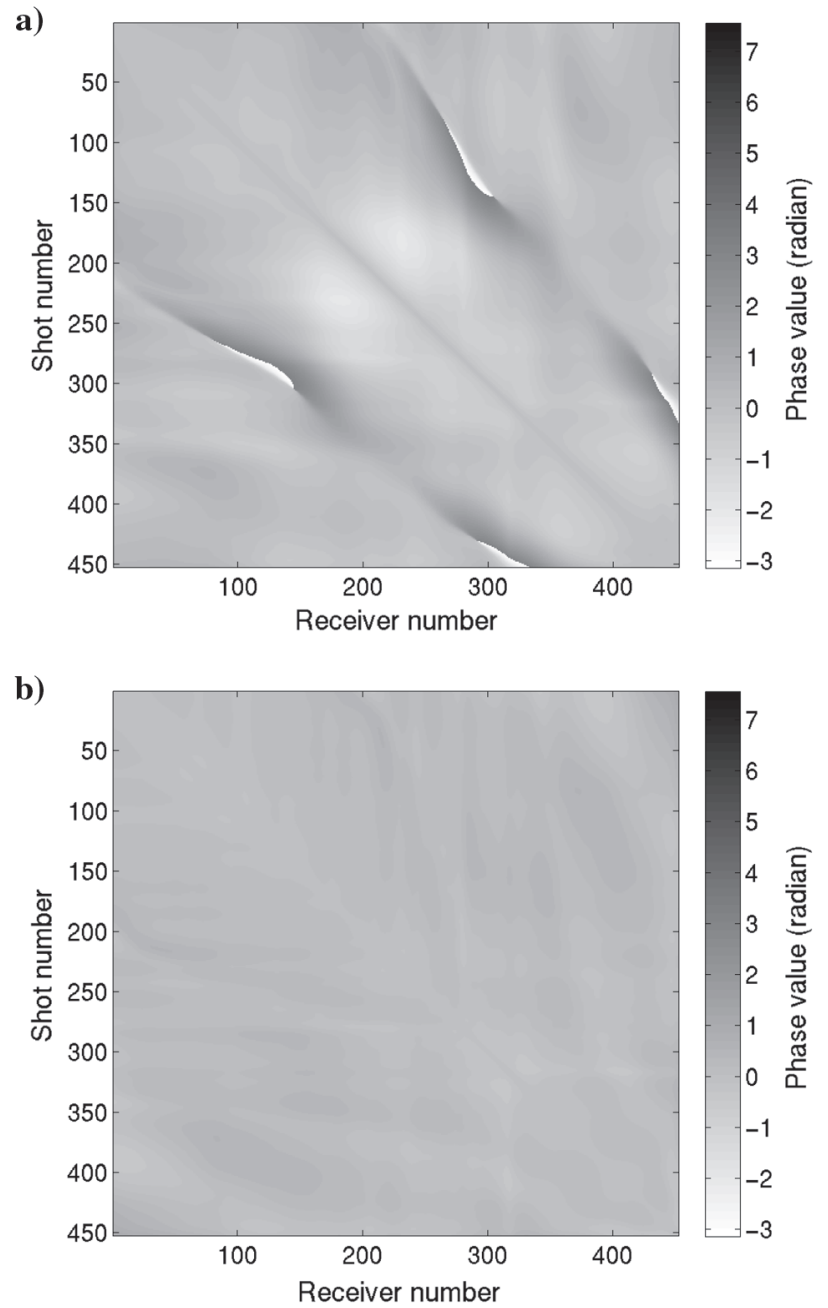

Figure 6. The (a) wrapped phase residual map and (b) unwrapped phase residual map at the final iteration.

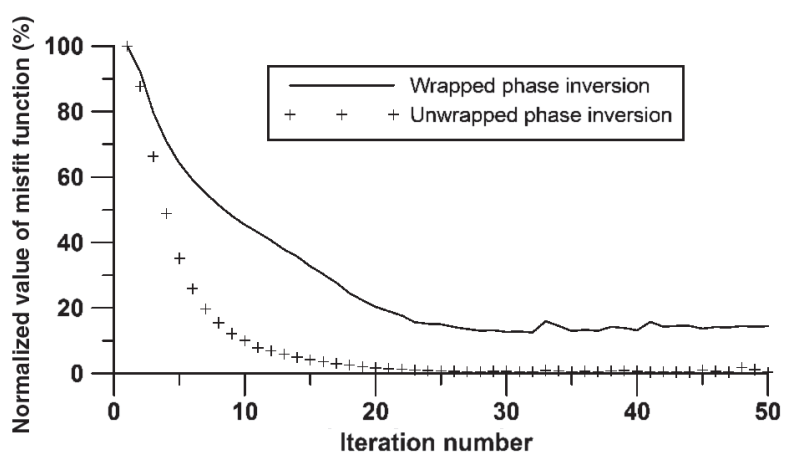

Figure 7. The history of the misfit functions of the wrapped and unwrapped phase inversion. All values of the misfit function are normalized by the value of the first iteration. 
not overlap. Because the phase unwrapping process is carried along a path line in a $2 \mathrm{D}$ phase map, it is straightforward to extend the unwrapping process to $3 \mathrm{D}$ phase cube for the $3 \mathrm{D}$ unwrapped phase inversion.

Figure 4 shows the original (wrapped) phase residual map with a damping factor of $30 \mathrm{~s}^{-1}$ and its unwrapped version, and also a comparison of profiles at the same shot numbers of 200 and 300 is shown (Figures $3 \mathrm{~d}$ and $4 \mathrm{a}$ are the same phase residual map with different scale ranges). We note that the original (wrapped) phase map is well-unwrapped through the unwrapping process. In our study, we invert the unwrapped phases in Figure $4 \mathrm{~b}$.

a)
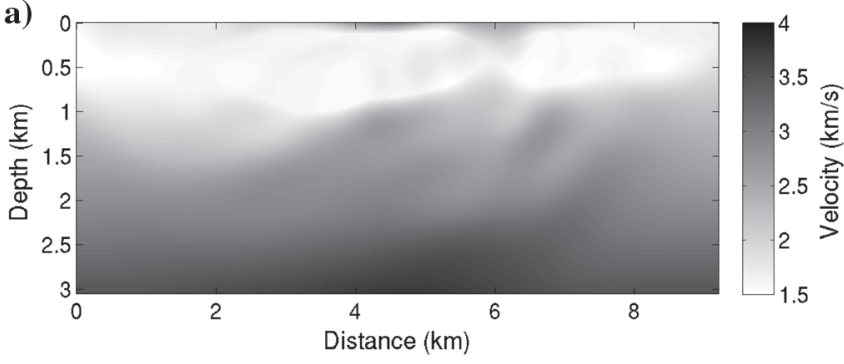

b)
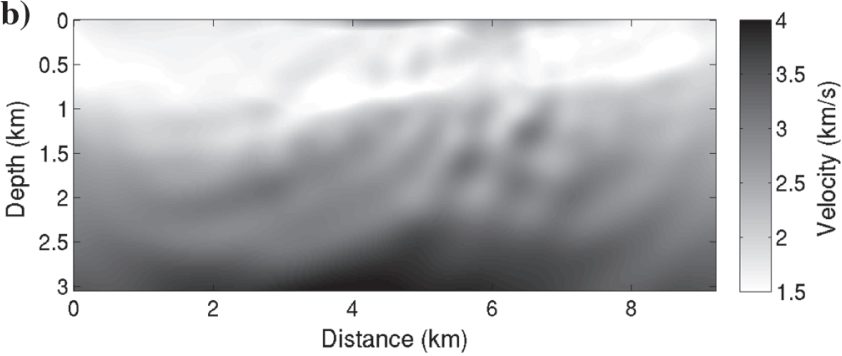

\section{Unwrapped phase inversion algorithm}

For the unwrapped phase inversion algorithm, we follow the logarithmic phase-only inversion theory of Min and Shin (2006) with the unwrapped phases. The objective function for the unwrapped phase inversion is the least-squares of the unwrapped phase residual, which is expressed for a single frequency as

$$
E=\sum_{i} \sum_{j} \frac{1}{2}\left[\mathrm{UW}\left(\theta_{i, j}^{u}-\theta_{i, j}^{d}\right)\right]^{2},
$$
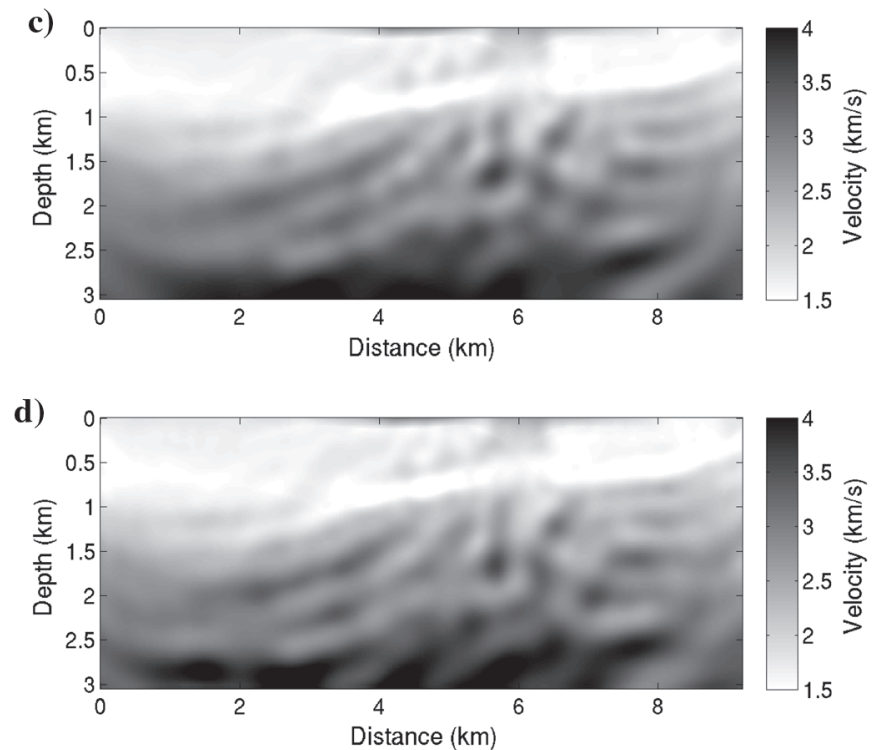

Figure 8. The inverted velocity models by the wrapped phase inversion with progressively reduced damping factors of (a) 5 , (b) 2 , (c) 1 , and (d) $0.2 \mathrm{~s}^{-1}$. Each inverted model is used as a new starting model for the wrapped phase inversion with a smaller damping factor.
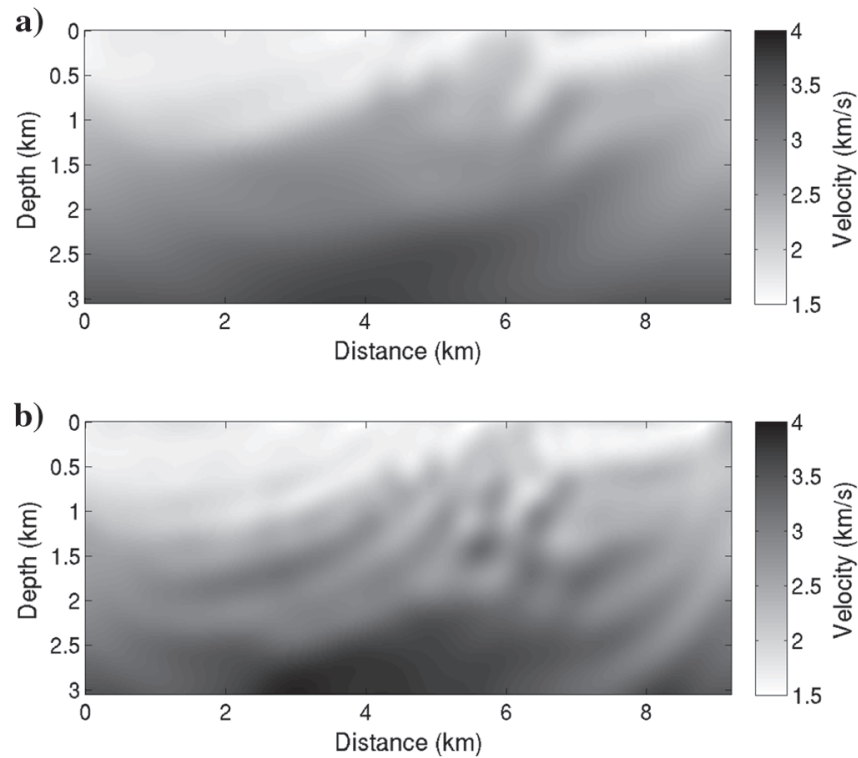
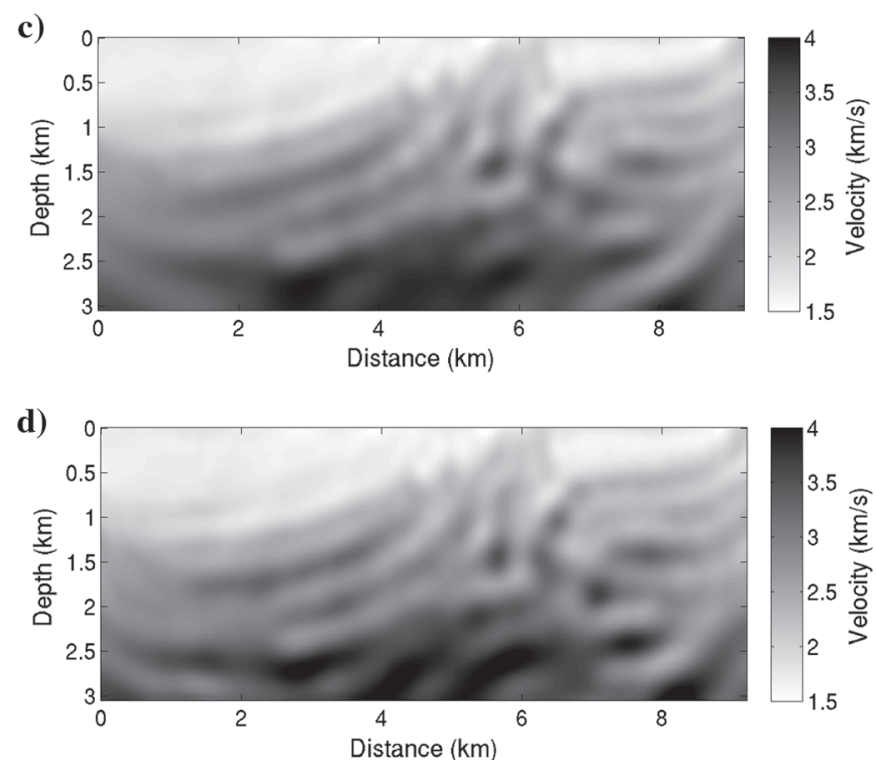

Figure 9. The inverted velocity models by the unwrapped phase inversion with progressively reduced damping factors of (a) 5 , (b) 2 , (c) 1 , and (d) $0.2 \mathrm{~s}^{-1}$. Each inverted model is used as a new starting model for the unwrapped phase inversion with a smaller damping factor. 
where $\mathrm{UW}()$ stands for the unwrapped version of phase. The gradient of the objective function with respect to the $k$ th model parameter $p_{k}$ is finally expressed in matrix and vector form as

$$
\frac{\partial E}{\partial p_{k}}=\operatorname{Im}\left\{\left(\mathbf{v}_{k}\right)^{T} \hat{\mathbf{S}}^{-1} \mathbf{r}_{u w}\right\},
$$

where $\mathbf{v}_{k}=-\left(\partial \hat{\mathbf{S}} / \partial p_{k}\right) \hat{\mathbf{u}}$, the element of $\mathbf{r}_{u w}$ is $\left[\operatorname{UW}\left(\theta_{i, j}^{u}-\right.\right.$ $\left.\left.\theta_{i, j}^{d}\right)\right] / \hat{u}_{i, j}$, and superscript $T$ stands for the transpose. The gradient expression in equation 10 is almost the same as that of conventional FWI except for the new residual vector $\mathbf{r}_{u w}$ (Pratt et al., 1998). On the other hand, FWI using the phase derivative, which is also free from the phase wrapping problem, requires five modeling steps (Choi and Alkhalifah, 2013) in one iteration, whereas we need only two modeling steps in the unwrapped phase inversion algorithm.

We scale the calculated gradient with the pseudo-Hessian (Shin et al., 2001) and use the conjugate-gradient method to update the model parameters. To obtain a proper step length, we can use a linesearch method but, for simplicity, we use a fixed-step length. We set the minimum and maximum velocities and constrain updated velocities within the bounds during the inversion.
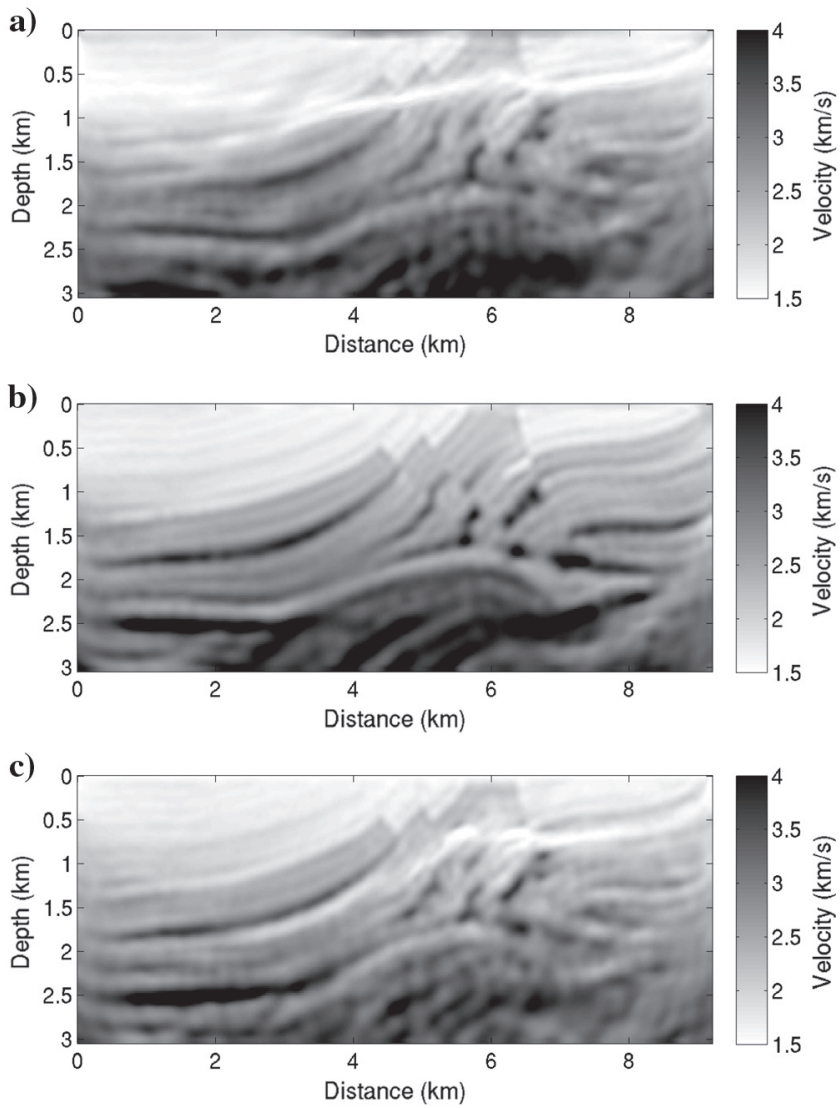

Figure 10 . The inverted velocity models by the subsequent conventional waveform inversion without damping for a frequency band of 3-7 Hz starting from (a) Figure 8d, (b) Figure 9d, and (c) the initial starting model in Figure 1b.
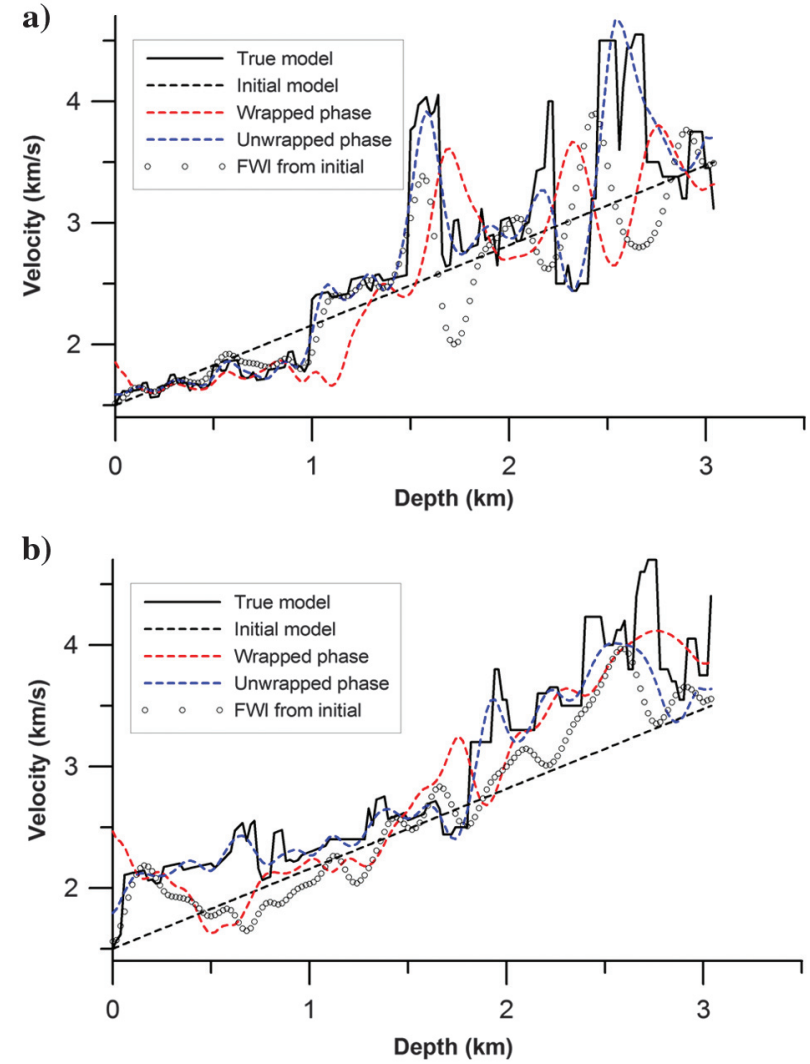

Figure 11. Depth profiles of the true model, initial model, and inverted models in Figure 10 at the distances of (a) 3 and (b) $6 \mathrm{~km}$.

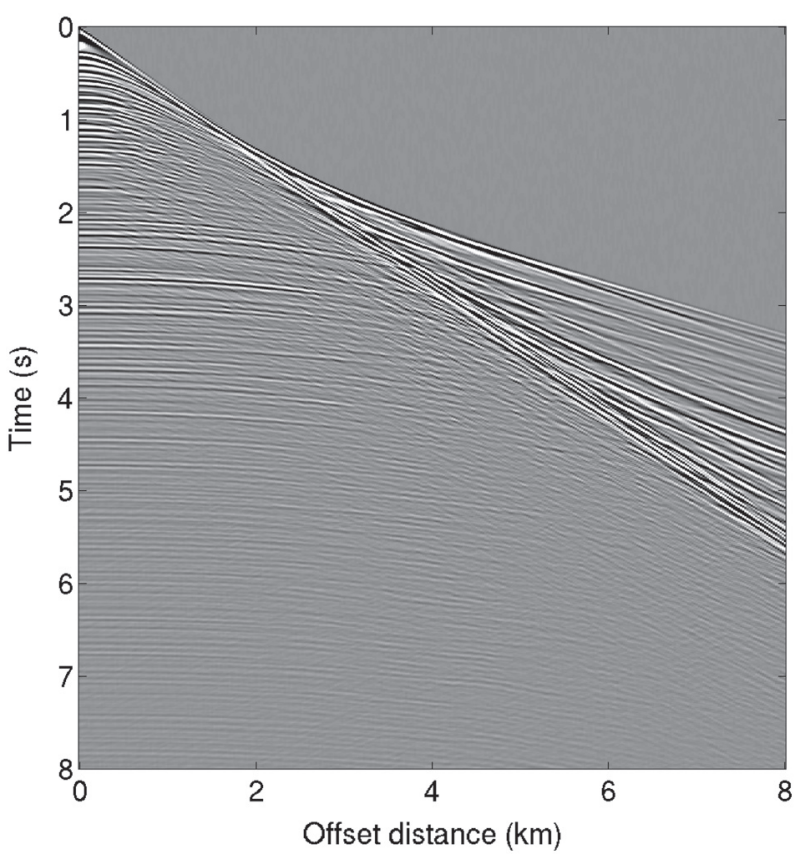

Figure 12. Representative seismogram of the SEG 2014 benchmark data set. 


\section{NUMERICAL EXAMPLES}

\section{Marmousi synthetic example}

We generate the synthetic data from the Marmousi model shown in Figure 1a and apply the unwrapped phase inversion to the synthetic data to validate our algorithm. The starting model for the inversion is a linearly increasing velocity model (Figure 1b). We assume that the frequency information less than $3 \mathrm{~Hz}$ is not available. The configuration of sources and receivers is the same as that of the examples of phase residual map in Figure 2. We regard the source wavelet to be known. We use the conjugate-gradient method for optimization and apply the Gaussian filter (Gaussian smoothing) to the calculated gradient (Virieux and Operto, 2009).

We apply the wrapped (original) phase inversion and our unwrapped phase inversion to the data of a single frequency of $3 \mathrm{~Hz}$ with a damping factor of $30 \mathrm{~s}^{-1}$. The phase residual maps at the first iteration for the wrapped and unwrapped phase inversion are the same as those in Figure $4 \mathrm{a}$ and $4 \mathrm{~b}$, respectively. The inverted models of both inversion methods after 50 iterations are shown in

a)

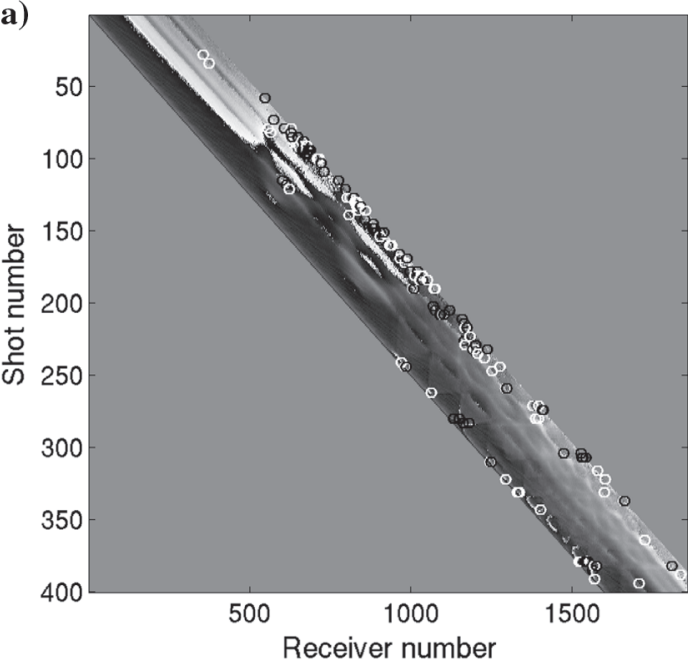

b)

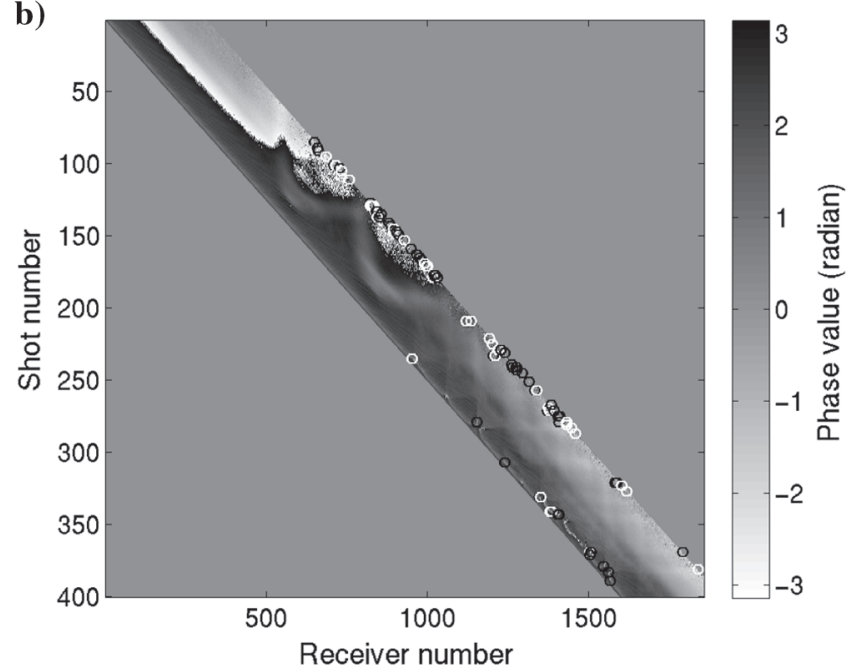

Figure 5. Because we apply a strong damping $\left(30 \mathrm{~s}^{-1}\right)$, the inverted model of the unwrapped phase inversion (Figure 5b) shows longwavelength features of the true model as the traveltime tomography does, whereas that of the wrapped phase inversion (Figure 5a) shows an inaccurate convergence because of the phase wrapping problem.

Figure 6 shows the phase residual maps of both phase inversion methods at the final iteration. The phase residual values of the unwrapped phase inversion (Figure 6b) decrease well at the final iteration and become close to zero, whereas the phase residual map of the wrapped phase inversion (Figure 6a) shows some high values and still has phase wrapping effects. Figure 7 shows the history of the misfit functions of the wrapped and unwrapped phase inversion. All values of the misfit function are normalized by the value at the first iteration. The misfit function of the unwrapped phase inversion decreases finally less than $1 \%$ of the initial value, whereas that of the wrapped phase inversion does not decrease less than $10 \%$ of the initial value. From Figures 6 and 7, we note that the unwrapped phase inversion algorithm for a single frequency of $3 \mathrm{~Hz}$ with a
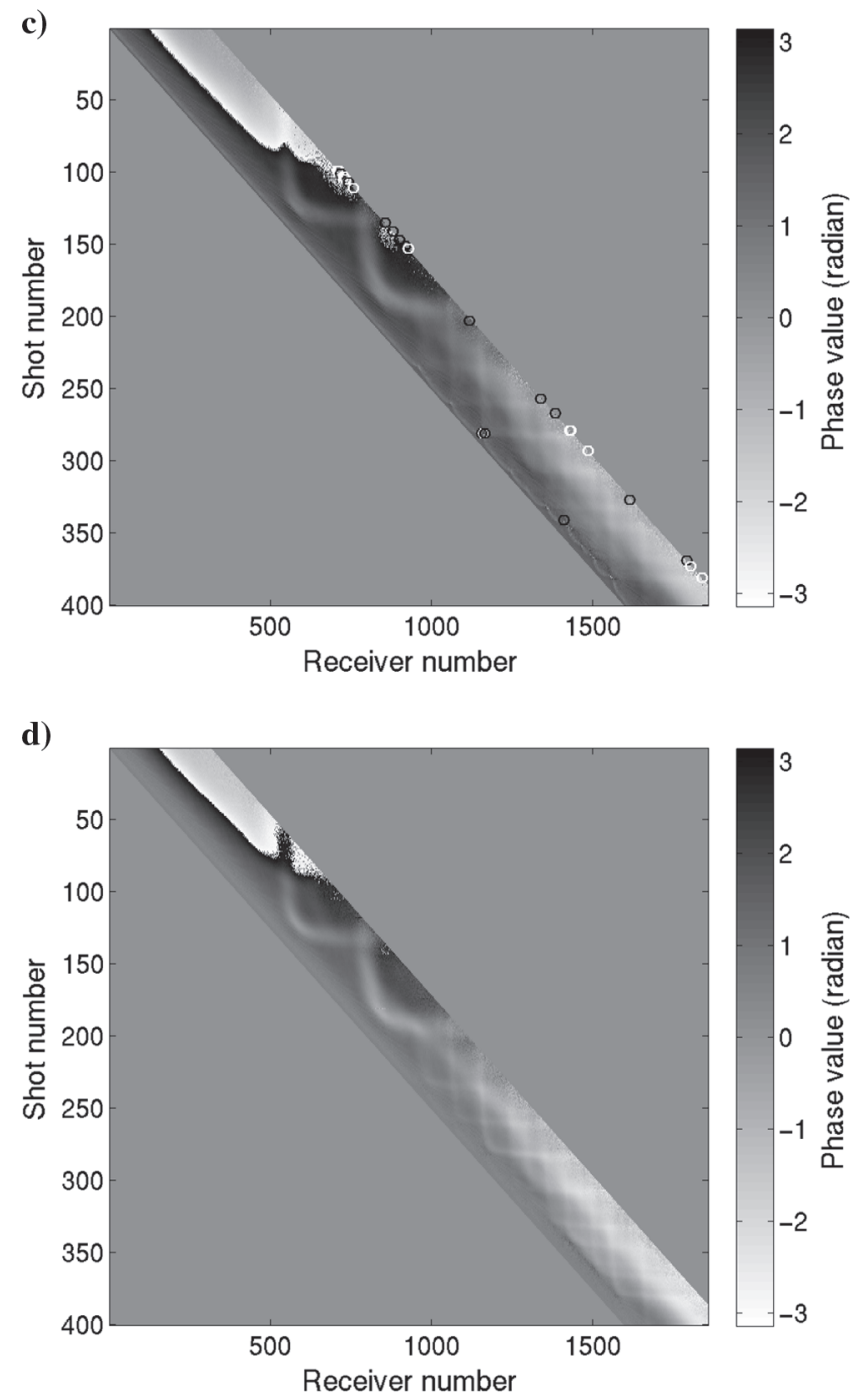

Figure 13. The phase residual maps at $3 \mathrm{~Hz}$ with damping factors of (a) 2, (b) 5, (c) 10, and (d) $30 \mathrm{~s}^{-1}$ with detected residues. The black and white circles indicate the detected plus and minus charge residues, respectively. 
damping factor of $30 \mathrm{~s}^{-1}$ provides a smooth convergent result, whereas the wrapped phase inversion does not.

In the next stage, we progressively reduce the damping factors as $5,2,1$, and $0.2 \mathrm{~s}^{-1}$ for the same frequency $(3 \mathrm{~Hz})$ data to increase the resolution of the inverted model. We perform the original (or wrapped) phase inversion for the reduced damping factors because the phase map at a somewhat low damping factor has residues and the inverted model of the unwrapped phase inversion with a strong damping has long enough wavelength features to be used as a good starting model for the inversion with a reduced damping. Figure 8 shows the inverted models starting from the wrapped phase inversion result (Figure 5a) with progressively reduced damping factors. The resolution of the inverted models increases as the damping factor decreases, but the inverted models still have some artifacts in the shallow part and incorrect structures in the deeper part caused by the phase wrapping effect. However, Figure 9 shows the inverted models starting from the unwrapped phase inversion result (Figure 5b) with progressively reduced damping factors. The inverted models have not only higher resolution but also reasonable structures without artifacts.

We finally perform the subsequent frequency-domain FWI without damping using the previous inverted models obtained with a damping factor of $0.2 \mathrm{~s}^{-1}$ (Figures $8 \mathrm{~d}$ and 9d) and the initial model (Figure $1 \mathrm{~b}$ ) as new starting models. The frequency band for sub-
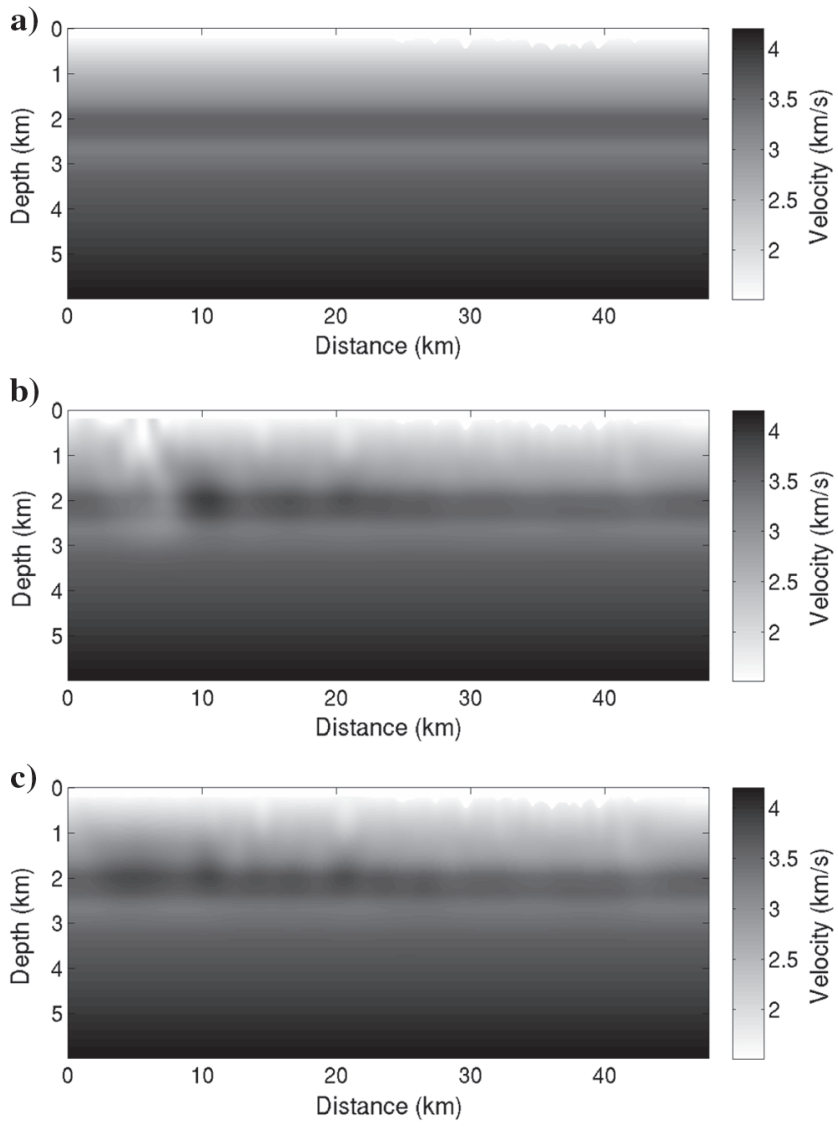

Figure 14. The (a) given initial model and inverted models by (b) the wrapped phase inversion and (c) the unwrapped phase inversion for $3 \mathrm{~Hz}$ data with damping factor of $30 \mathrm{~s}^{-1}$. sequent frequency-domain FWI is 3-7 Hz. We set three subgroups of frequencies: $3-5,4-6$, and 5-7 Hz with a frequency interval of $0.125 \mathrm{~Hz}$. We calculate gradients of all frequencies in a subgroup and sum those to update the velocity model. We start inversion with the lowest frequency subgroup and progressively move to the higher frequency subgroup.

Figure 10 shows the inverted models obtained from the subsequent frequency-domain FWI. Figure 11 shows the depth profiles of the true model, initial starting model, and the inverted models at the distances of 3 and $6 \mathrm{~km}$. The inverted model starting from the unwrapped phase inversion result (Figure 10b) and its depth profiles (Figure 11) are comparable with the true model, whereas those starting from the wrapped phase inversion results (Figure 10a) and the initial staring model (Figure 10c) exhibit incorrect structures caused by the phase wrapping problem.

We note that the unwrapped phase inversion with strong damping provides a convergent long-wavelength structure model without low-frequency information. This long-wavelength model can be used as a good starting model for the inversion with a reduced damping and conventional FWI.

\section{SEG 2014 benchmark data example}

The benchmark data set is 2D marine isotropic elastic synthetic data that includes noise. We downloaded the benchmark data from http://s3.amazonaws.com/open.source.geoscience/open_data/seg_ workshop_fwi/seg_workshop_fwi.html. An initial velocity model was also provided. The true velocity model is not yet released, and only a single velocity profile of the true model at a well log was provided; thus, this inversion is a true blind test. The benchmark data have a maximum offset length of $8 \mathrm{~km}$ and a recording time of 8 s. Figure 12 shows a representative shot gather of the SEG 2014 benchmark data. Because it is a computational burden to deal with all of the shot gathers available, we select every fourth shot gather; thus, only 400 shot gathers are treated in this example. The shot interval of the selected shot gathers is $100 \mathrm{~m}$, and the receiver interval is $25 \mathrm{~m}$. In the data, frequency information less than $3 \mathrm{~Hz}$ is not available; thus, we start the inversion from a frequency of $3 \mathrm{~Hz}$. We estimate the source wavelet through the unwrapped phase inversion (Bednar et al., 2007). Because the data are contaminated with noise, we perform the first-arrival picking and mute the data prior to the first-arrival events to allow us to apply exponential damping.

We apply several levels of exponential damping to the observed and modeled data from the given initial model and construct phase residual maps with detected residues for $3 \mathrm{~Hz}$ data (Figure 13). For damping factors of 2,5 , and $10 \mathrm{~s}^{-1}$, the phase residual maps include residue points (Figure 13a-13c); thus, the phase unwrapping process cannot be applied to the phase residual map. On the other hand, the phase residual map for a damping factor of $30 \mathrm{~s}^{-1}$ has no residue point (Figure 13d); thus, it can be unwrapped. The phase residual map for a damping factor of $30 \mathrm{~s}^{-1}$ (Figure 13d) shows the phase wrapping phenomena for the first 100 shot gathers and no phase wrapping for the other shot gathers, which means the given initial model is close enough to the true model in the right part and quite far from the true one in the left part. We finally unwrap the phase residual map for a damping factor of $30 \mathrm{~s}^{-1}$ and invert the unwrapped phases.

Even though the benchmark data are elastic data, we use an acoustic-based frequency-domain modeling technique for the inver- 
sion. We apply the wrapped and unwrapped phase inversion to the benchmark data for $3 \mathrm{~Hz}$ with a damping factor of $30 \mathrm{~s}^{-1}$ and compare the results. Figure 14 shows the given initial model and inverted models by the wrapped and unwrapped phase inversion. The inverted model by the unwrapped phase inversion shows some improvements in the shallow left side of the model (Figure 14c), whereas that by the wrapped phase inversion includes an artifact in the same area (Figure 14b). Figure 15 shows the phase residual maps of the wrapped and unwrapped phase inversion from the given initial model (first iteration) and final inverted models (final iteration). For clear comparison, we construct the phase residual maps using only the first 200 selected shot gathers. We note that the phase residual values of the unwrapped phase inversion greatly decrease after the final iteration (Figure 15b and 15d), whereas the final phase residual map of the wrapped phase inversion still has large values for a shot range of 1-45 (Figure 15c). Figure 16 shows the history of the misfit function of the wrapped and unwrapped phase inversion, which also demonstrates the good convergence of the unwrapped phase inversion for the benchmark data.
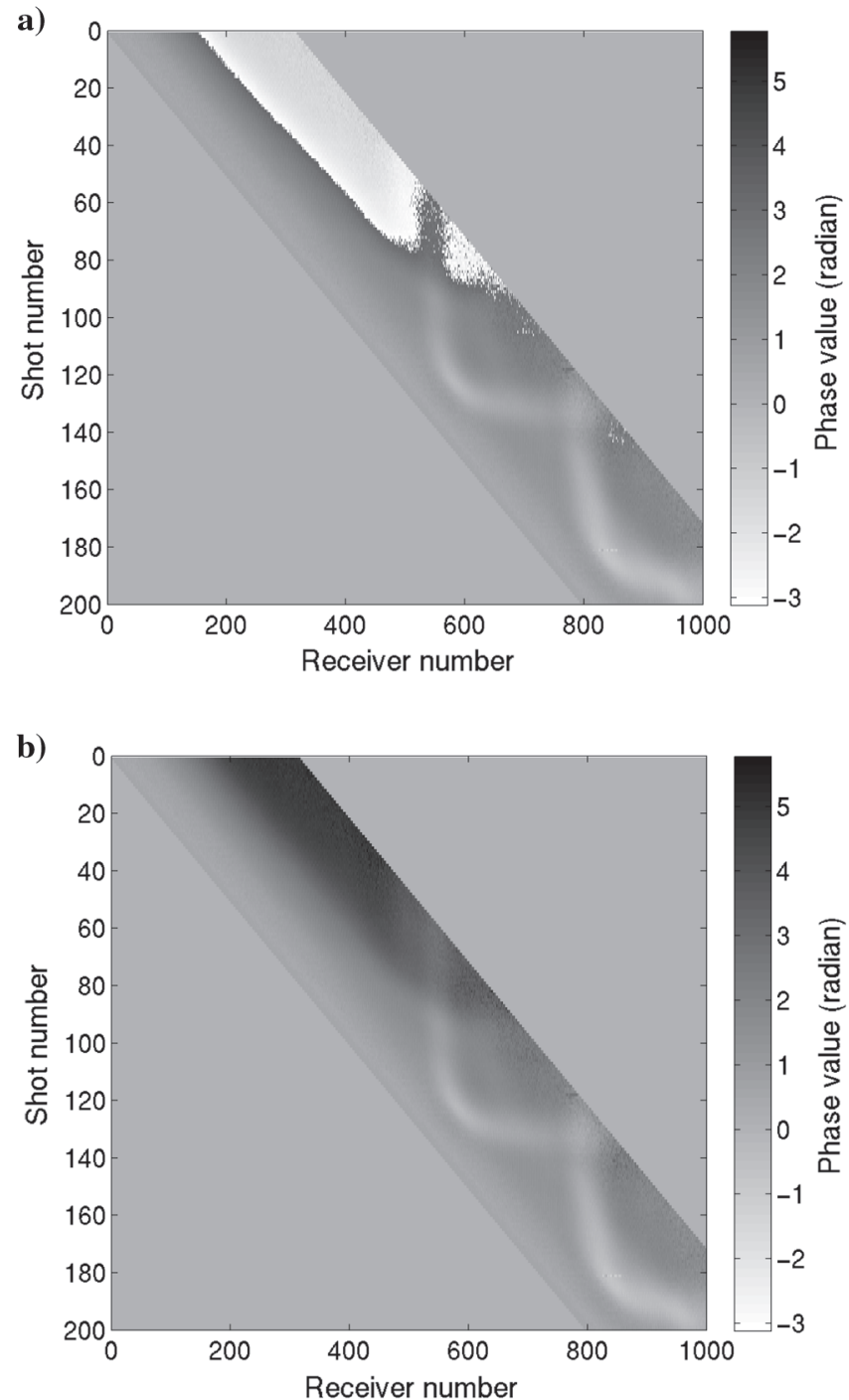

We carry out the subsequent frequency-domain FWI without damping for a frequency band of $3-13 \mathrm{~Hz}$ starting from the given initial model (Figure 14a) and the final inverted models of the

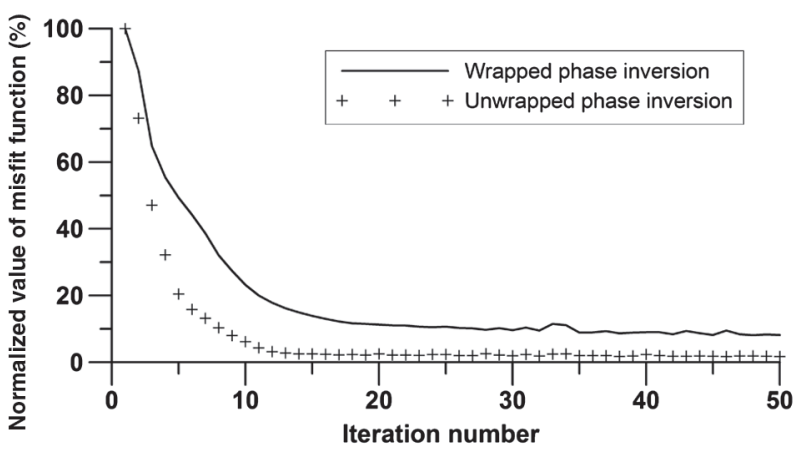

Figure 16. The history of the misfit functions of the wrapped and unwrapped phase inversion for the SEG 2014 benchmark data set. All values of the misfit function are normalized by the value of the first iteration.
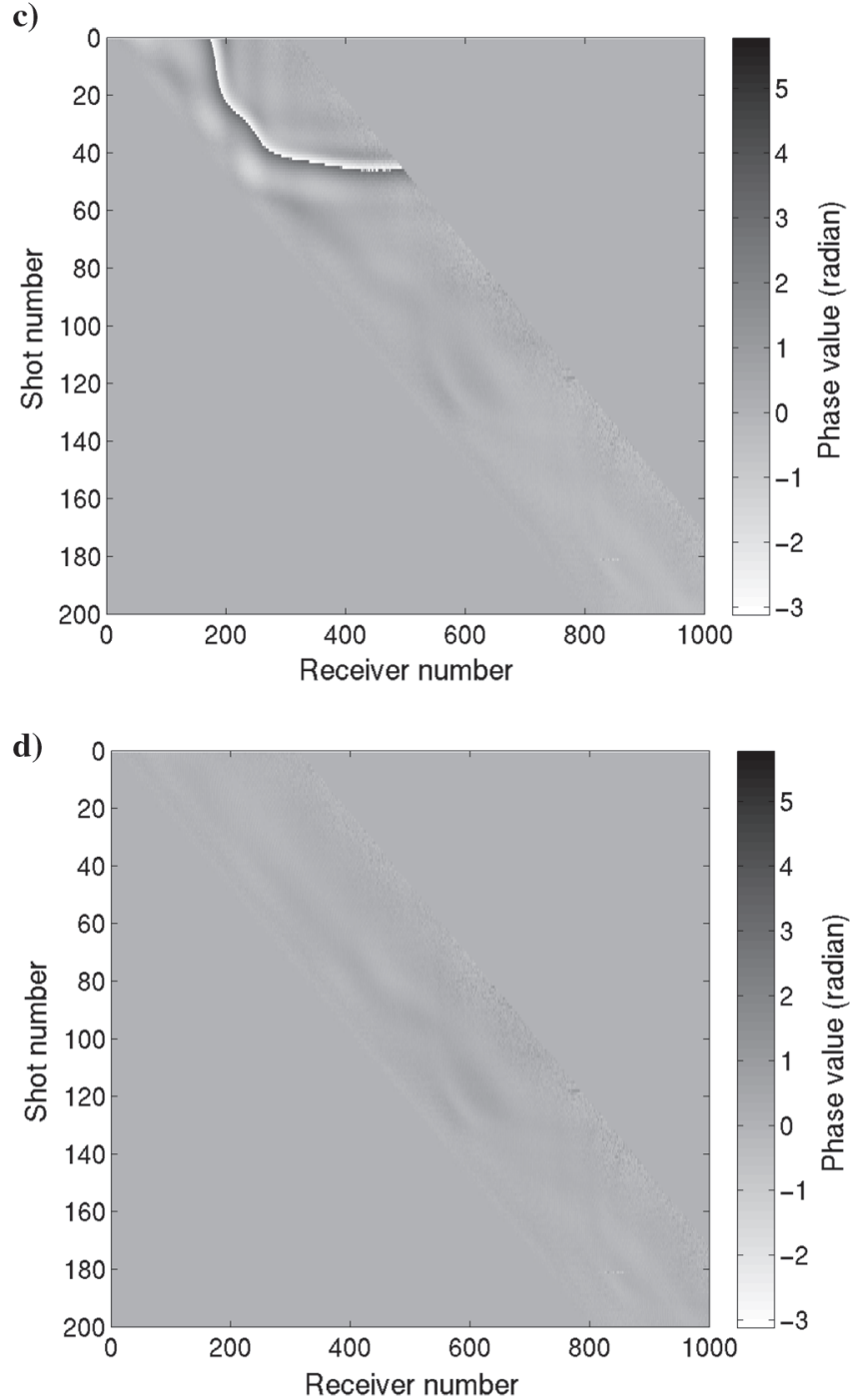

Figure 15. The wrapped and unwrapped phase residual maps from the (a and b) given initial model and (c and d) final inverted models in Figure 14, respectively. 
wrapped and unwrapped phase inversions (Figure 14b and 14c). We set three subgroups of frequencies: $3-7,6-10$, and 9-13 Hz with a frequency interval of $0.25 \mathrm{~Hz}$, and we use the same strategy with the previous example to update velocity model.

Figure 17 shows the inverted velocity models obtained by the subsequent frequency-domain FWI without damping. All inverted
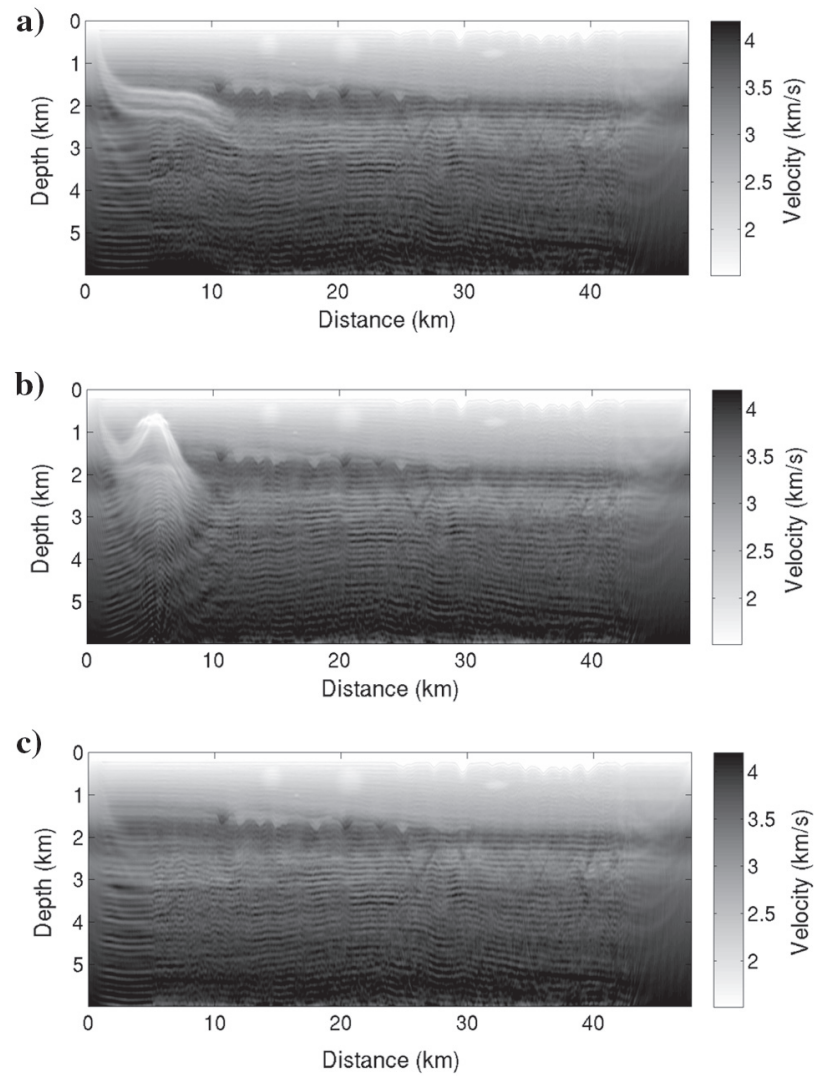

Figure 17 . The inverted velocity models by the subsequent conventional waveform inversion for a frequency band of 3-13 Hz starting from the (a) given initial model in Figure 14a and (b and c) the final inverted models of the wrapped and unwrapped phase inversion in Figure $14 \mathrm{~b}$ and $14 \mathrm{c}$.

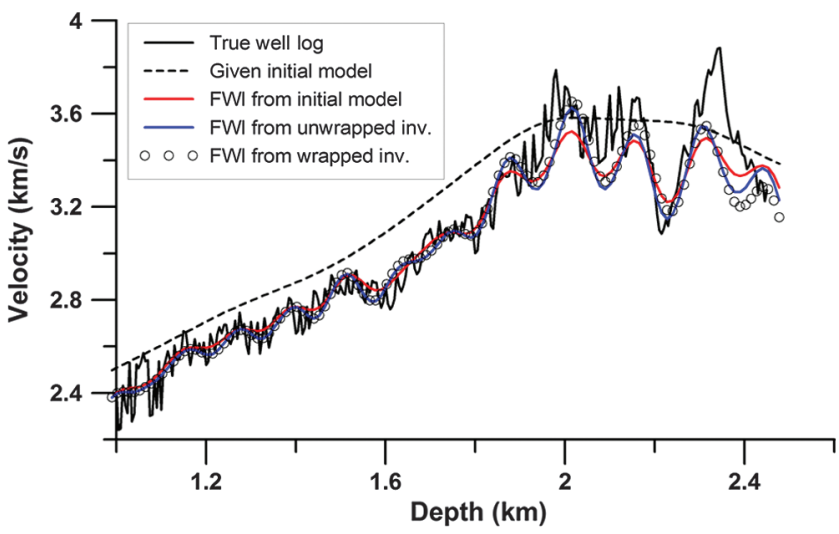

Figure 18. Comparison of the given well-log velocity values and the depth profiles of the initial and inverted models in Figure 17 at a distance of $39.375 \mathrm{~km}$.
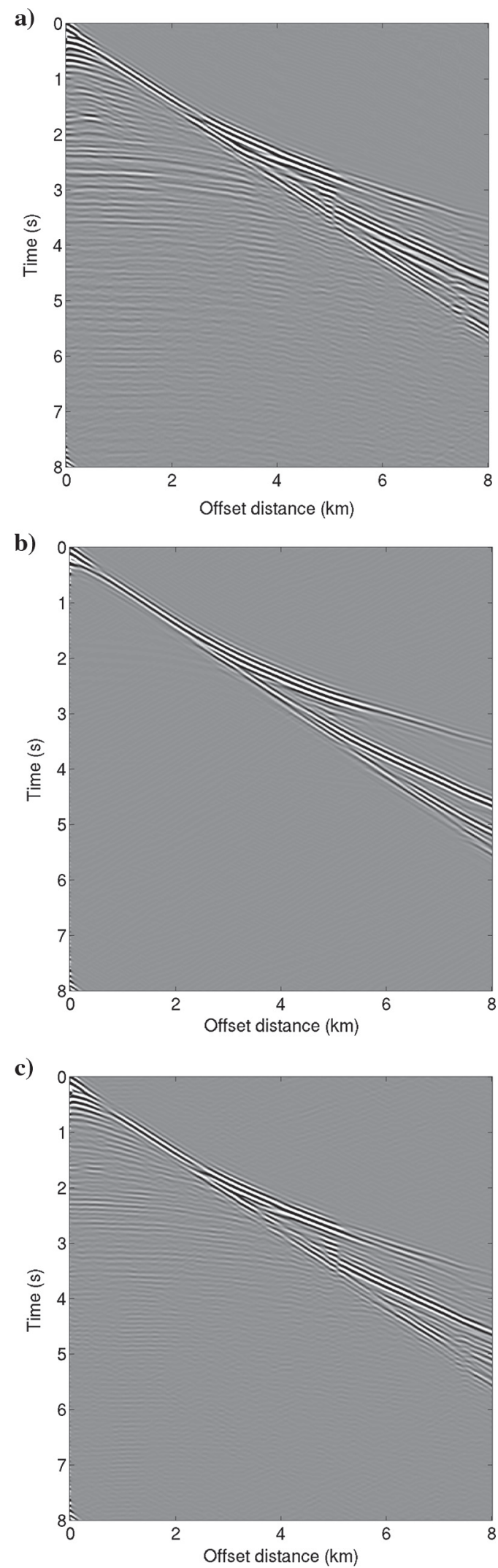

Figure 19. The time-domain (a) observed seismogram and modeled seismograms generated from the (b) unwrapped phase inversion result in Figure 14c and (c) subsequent FWI result in Figure 17c. The seismograms are filtered with a band-pass of $3-13 \mathrm{~Hz}$. 
models show three lens-shaped low-velocity structures near the surface and a low-velocity layer at an approximate depth of $2.5 \mathrm{~km}$. The inverted model starting from the unwrapped phase inversion result shows a reasonably continuous image (Figure 17c), whereas those starting from the given initial model and wrapped phase inversion result have an artifact-looking image in the left side of the model (Figure 17a and 17b). We compare the true velocity profile with the depth profiles of the initial and final inverted models (Figure 18). The only subsurface truth information provided was a single velocity profile with a depth range of $1-2.45 \mathrm{~km}$. The horizontal location of the true velocity profile is $39.375 \mathrm{~km}$. The velocity profiles of all final inverted models in Figure 17 show good convergences to the true velocity profile. Figures 17 and 18 also prove that the given initial model (Figure 14a) is close enough to the true model in the right part and quite far from the true one in the left part.

As a final quality-control step, we generated the time-domain modeled seismograms from the inverted models of the unwrapped phase inversion in Figure 14c and subsequent FWI in Figure 17c and compare these with the observed seismogram. The observed and modeled seismograms are filtered with a band-pass of 3 $13 \mathrm{~Hz}$. The modeled seismogram generated from the unwrapped phase inversion result exhibits good convergence only for the direct, transmitted, and diving waves in the observed seismogram and no reflected waves (Figure 19b), whereas that from the subsequent FWI result also includes rough features of the reflected waves in the observed seismogram (Figure 19c). Figure 20 shows the comparison of some selected traces of the observed seismogram (black line) in Figure 19a and the modeled seismogram (red line) in Figure $19 \mathrm{c}$ for several offset distances. We display the traces till $4.5 \mathrm{~s}$ for clear comparison. The modeled wavefield converges well with the observed wavefield especially around the first-arrival events.

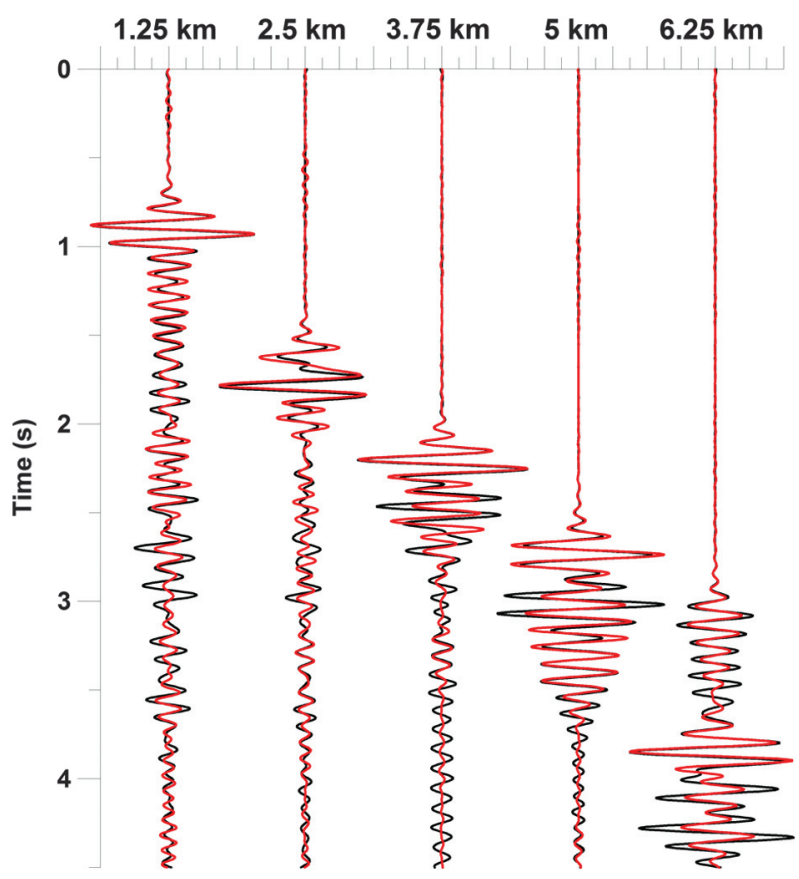

Figure 20. Comparison of the traces of the observed seismogram in Figure 19a (black line) and modeled seismogram in Figure 19c (red line) at offset distances of $1.25,2.5,3.75,5$, and $6.25 \mathrm{~km}$.
From the examples, we note that our unwrapped phase inversion provides a long-wavelength structure model for the benchmark data set without low-frequency information, which can be used as a good starting model for conventional FWI with an increased frequency band.

\section{DISCUSSION}

Strong damping attenuates later arrival events (mostly reflected waves) and reserves only information of the first arrival event (e.g., transmitted waves, diving waves, etc.). The unwrapped phase inversion with a strong damping thus inverts the phases of the first arrival event, which is similar to the first-arrival traveltime tomography. However, conventional traveltime tomography algorithms use the raypath or banana-doughnut-shaped sensitivity kernels, whereas the full-sensitivity kernel is used in unwrapped phase inversion with strong damping (Van Leeuwen and Mulder, 2010; Djebbi and Alkhalifah, 2013). As we reduce the damping factor, the phase of the wavefield includes more information of later arrival events (reflected waves), and thus inversion with a reduced damping can describe the deeper part of the model and provide a higher resolution image.

When strong damping is applied, noise prior to the first-arrival event is strongly amplified, and therefore it should be removed through windowing based on first-arrival picking. We should also consider the precision level of computing when applying a high damping factor to get a stable and accurate value of the damped wavefield.

\section{CONCLUSIONS}

The phase wrapping problem is a big obstacle to the successful implementation of FWI when low-frequency information is not available. In this study, we have suggested an inversion algorithm using unwrapped phases, especially for somewhat high frequency data. We first constructed a 2D phase residual map, and then we unwrapped the phase map. However, if the phase map includes several residues caused usually by complex reflections at somewhat high frequencies, it is difficult to unwrap the phase map, and the unwrapping process usually does not provide a unique solution. In this case, we applied a strong exponential damping to the wavefield to remove the residues in the phase residual map. We unwrapped the phase residual map at a high damping factor, and we then inverted the unwrapped phases. The unwrapped phase inversion algorithm shares the same back-propagation algorithm as in conventional FWI except for the new residual vector. We progressively reduced the damping factor to obtain higher resolution gradients. Numerical examples showed that our unwrapped phase inversion provided a convergent long-wavelength structure model without low-frequency information. This inverted model can be used as a good starting model for the inversion with reduced damping and conventional FWI.

\section{ACKNOWLEDGMENTS}

We are grateful to King Abdullah University of Science and Technology for financial support. We thank Chevron for providing the SEG 2014 benchmark data. We also thank S. Operto, G. Pratt, and D.-J. Min for their review and helpful comments. 


\section{REFERENCES}

Bednar, J. B., C. Shin, and S. Pyun, 2007, Comparison of waveform inversion. Part 2: Phase approach: Geophysical Prospecting, 55, 465-475, doi: 10.1111/j.1365-2478.2007.00618.x.

Brenders, A. J., and R. G. Pratt, 2007, Full waveform tomography for lithospheric imaging: Results from a blind test in a realistic crustal model: Geophysical Journal International, 168, 133-151, doi: 10.1111/j.1365246X.2006.03156.x.

Brossier, R., S. Operto, and J. Virieux, 2009, Seismic imaging of complex onshore structures by 2D elastic frequency-domain full-waveform inversion: Geophysics, 74, no. 6, WCC105-WCC118, doi: 10.1190/1.3215771.

Bunks, C., F. M. Saleck, S. Zaleski, and G. Chavent, 1995, Multiscale seismic waveform inversion: Geophysics, 60, 1457-1473, doi: 10.1190/1 1443880 .

Choi, Y., and T. Alkhalifah, 2011, Frequency-domain waveform inversion using the unwrapped phase: 81st Annual International Meeting, SEG, Expanded Abstracts, 2576-2580.

Choi, Y., and T. Alkhalifah, 2013, Frequency-domain waveform inversion using the phase derivative: Geophysical Journal International, 195, 19041916, doi: 10.1093/gji/ggt351.

Djebbi, R., and T. Alkhalifah, 2013, Wave equation tomography using the unwrapped phase - Analysis of the traveltime sensitivity kernels: 75th Annual International Conference and Exhibition, EAGE, Extended Abstracts, Tu0707.

Flynn, T. J., 1996, Consistent 2-D phase unwrapping guided by a quality map: Proceedings of IEEE conference on Geoscience and Remote Sensing Symposium, 2057-2059.

Ghiglia, D. C., and M. D. Pritt, 1998, Two-dimensional phase unwrapping: Theory, algorithms, and software: John \& Sons Inc.

Goldstein, R. M., H. A. Zebker, and C. L. Werner, 1988, Satellite radar interferometry: Two dimensional phase unwrapping: Radio Science, 23, 713720, doi: 10.1029/RS023i004p00713.

Itoh, K., 1982, Analysis of the phase unwrapping problem: Applied Optics, 21, 2470-2470, doi: 10.1364/AO.21.002470.

McGowan, R., and R. Kuc, 1982, A direct relation between a signal time series and its unwrapped phase: IEEE Transactions on Acoustics, Speech and Signal Processing, 30, 719-726, doi: 10.1109/TASSP.1982.1163965.

Min, D. J., and C. Shin, 2006, Refraction tomography using a waveforminversion back-propagation technique: Geophysics, 71, no. 3, R21-R30, doi: $10.1190 / 1.2194522$.

Oh, J. W., and D. J. Min, 2013, Weighting technique using backpropagated wavefields incited by deconvolved residuals for frequency-domain elastic full waveform inversion: Geophysical Journal International, 194, 322347, doi: $10.1093 /$ gji/ggt107.
Oppenheim, A. V., and R. W. Schafer, 1975, Digital signal processing: Prentice Hall Inc.

Pratt, R. G., C. Shin, and G. J. Hicks, 1998, Gauss-Newton and full Newton methods in frequency domain seismic waveform inversion: Geophysical Journal International, 133, 341-362, doi: 10.1046/j.1365-246X.1998 .00498.x.

Shah, N. K., M. R. Warner, J. K. Washbourne, L. Guasch, and A. P. Umpleby, 2012, A phase-unwrapped solution for overcoming a poor starting model in full-wavefield inversion: 74th Annual International Conference and Exhibition, EAGE, Extended Abstracts, P014.

Shin, C., and Y. H. Cha, 2008, Waveform inversion in the Laplace domain: Geophysical Journal International, 173, 922-931, doi: 10.1111/j.1365246X.2008.03768.x.

Shin, C., and Y. H. Cha, 2009, Waveform inversion in the Laplace-Fourier domain: Geophysical Journal International, 177, 1067-1079, doi: 10 .1111/j.1365-246X.2009.04102.x.

Shin, C., S. Jang, and D. J. Min, 2001, Improved amplitude preservation for prestack depth migration by inverse scattering theory: Geophysical Prospecting, 49, 592-606, doi: 10.1046/j.1365-2478.2001.00279.x.

Shin, C., and D. J. Min, 2006, Waveform inversion using a logarithmic wavefield: Geophysics, 71, no. 3, R31-R42, doi: 10.1190/1.2194523.

Shin, C., D. J. Min, K. J. Marfurt, H. Y. Lim, D. Yang, Y. Cha, S. Ko, K. Yoon, T. Ha, and S. Hong, 2002, Traveltime and amplitude calculations using the damped wave solution: Geophysics, 67, 1637-1647, doi: 10 $.1190 / 1.1512811$.

Steiglitz, K., and B. Dickinson, 1982, Phase unwrapping by factorization: IEEE Transactions on Acoustics, Speech, and Signal Processing, 30, 984991, doi: 10.1109/TASSP.1982.1163975.

Su, X., and W. Chen, 2004, Reliability-guided phase unwrapping algorithm: A review: Optics and Lasers in Engineering, 42, 245-261, doi: 10.1016/j .optlaseng.2003.11.002.

Treitel, S., G. A. Sitton, J. W. Fox, and C. S. Burrus, 2006, Factoring highdegree polynomials with applications to geophysics: The Leading Edge, 25, 1216-1219, doi: 10.1190/1.2360607.

Tribolet, J. M., 1977, A new phase unwrapping algorithm: IEEE Transactions on Acoustics, Speech and Signal Processing, 25, 170-177, doi: 10 .1109/TASSP.1977.1162923.

Van Leeuwen, T., and W. A. Mulder, 2010, A correlation-based misfit criterion for wave-equation traveltime tomography: Geophysical Journal International, 182, 1383-1394, doi: 10.1111/j.1365-246X.2010.04681.x.

Virieux, J., and S. Operto, 2009, An overview of full-waveform inversion in exploration geophysics: Geophysics, 74, no. 6, WCC1-WCC26, doi: 10 $.1190 / 1.3238367$ 